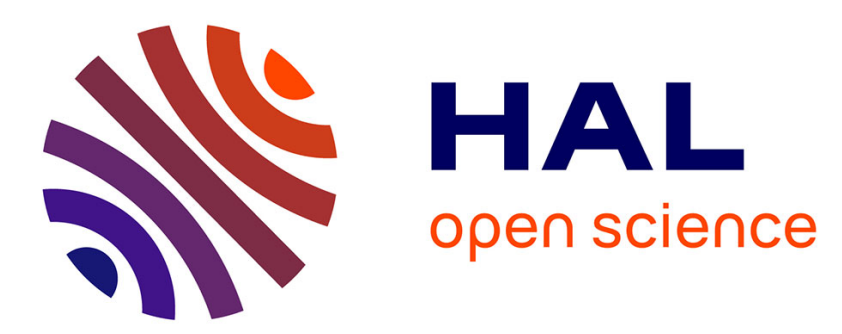

\title{
A complex version of the Cahn-Hilliard equation for grayscale image inpainting
}

Laurence Cherfils, Hussein Fakih, Alain Miranville

\section{To cite this version:}

Laurence Cherfils, Hussein Fakih, Alain Miranville. A complex version of the Cahn-Hilliard equation for grayscale image inpainting. 2015. hal-01200619

\section{HAL Id: hal-01200619 \\ https://hal.science/hal-01200619}

Preprint submitted on 16 Sep 2015

HAL is a multi-disciplinary open access archive for the deposit and dissemination of scientific research documents, whether they are published or not. The documents may come from teaching and research institutions in France or abroad, or from public or private research centers.
L'archive ouverte pluridisciplinaire HAL, est destinée au dépôt et à la diffusion de documents scientifiques de niveau recherche, publiés ou non, émanant des établissements d'enseignement et de recherche français ou étrangers, des laboratoires publics ou privés. 


\title{
A COMPLEX VERSION OF THE CAHN-HILLIARD EQUATION FOR GRAYSCALE IMAGE INPAINTING
}

\author{
LAURENCE CHERFILS ${ }^{1}$, HUSSEIN FAKIH $^{2}$, AND ALAIN MIRANVILLE ${ }^{2}$
}

\begin{abstract}
Our aim in this article is to propose a generalization of the BertozziEsedoglu-Gillette-Cahn-Hilliard equation, introduced for binary image inpainting, for grayscale image inpainting. In particular, we consider the solution to the corresponding Cahn-Hilliard inpainting model as a complex valued function. We are interested in the study of the well-posedness and of the asymptotic behavior, in terms of finitedimensional attractors, of the associated dynamical system. We have to face two major difficulties here. The first one comes from the fact that we no longer have the conservation of mass, i.e., of the spatial average of the order parameter $u$, contrary to the classical Cahn-Hilliard equation. The second one is due to the estimates on the nonlinear terms, combined with the fact that the order parameter $u$ is complex valued. We finally give numerical simulations which confirm and extend previous ones on the efficiency of the binary model.
\end{abstract}

\section{INTRODUCTION}

Image inpainting involves filling in parts of an image or a video from the surrounding area. It is essentially some type of interpolation. Its applications include restoration of old paintings by museum artists [31], removing scratches from old photographs [9], altering scenes in photographs [46], and restoration of motion pictures [49].

The work of Bertalmio et al. in [4] is very important, as it proposed a new direction in image inpainting by considering PDE's models. In particular, there, the authors proposed boundary conditions for PDE's image inpainting models. These boundary conditions consist of the constant grayscale image intensity and the direction of the isophote vectors at the boundary of the inpainting region. The isophote vector $\nabla^{\perp} u$ is the orthogonal gradient vector (e.g., in $\mathbb{R}^{2}, \nabla^{\perp} u=\left(-\frac{\partial u}{\partial y}, \frac{\partial u}{\partial x}\right)$ ).

Furthermore, Bertalmio et al. proposed in [3] a new PDE's model for image inpainting based on the Navier-Stokes equations. More precisely, the idea is to use a reformulation of the Navier-Stokes equations with an anisotropic term (to keep the no slip boundary conditions) and, of course, a fidelity term (such a term is essential in image inpainting, as it forces the solution to the PDE's to stay close to the original image outside the inpainting region).

Esedoglu and Shen [33] proposed two PDE's models for image inpainting based on the Mumford-Shah model. The first one is obtained by a simple modification of the fidelity term, while the second one is obtained by considering the Euler's elastic approximation

2010 Mathematics Subject Classification. 35B45, 35K55, 35B40, $68 \mathrm{U} 10$.

Key words and phrases. Cahn-Hilliard equation, grayscale image inpainting, well-posedness, finitedimensional attractors, simulations. 
(other PDE's inpainting models can be found in, e.g., [1], [14], [15], [16], [32], [39], [40], [41], [54], and [65]).

A simplified version of the Euler's elastic model is the Ginzburg-Landau energy. In particular, the Cahn-Hilliard equation can be derived as an $H^{-1}$-gradient flow of the Ginzburg-Landau energy, as proved by Fife [37].

The Cahn-Hilliard equation plays an important role in materials science and describes phase separation processes. This can be observed, e.g., when a binary alloy is cooled down sufficiently. One then observes a partial nucleation (i.e., the apparition of nucleides in the material) or a total nucleation, the so-called spinodal decomposition: the material quickly becomes inhomogeneous, forming a fine-grained structure in which each of the two components appears more or less alternatively. In a second stage, which is called coarsening and occurs at a slower time scale, these microstructures coarsen. Such phenomena play an essential role in the mechanical properties of the material, e.g., strength. We refer the reader to, e.g., [11], [12], [20], [29], [48], [50], [52], [53], [59], and [60] for more details.

It is also interesting to note that the Cahn-Hilliard equation, or some of its variants, is relevant in other contexts, in which phase separation and coarsening/clustering processes can be observed or come into play. We can mention, for instance, population dynamics (see [23]), bacterial films (see [47]), wound healing and tumor growth (see [21], [36], [45], [55], and [56]), thin films (see [61] and [67]), image processing and inpainting (see [5], [6], [10], [13], [17], and [25]), and even the rings of Saturn (see [68]) and the clustering of mussels (see [51]).

Binary image inpainting with fourth-order PDE's. Bertozzi, Esedoglu, and Gillette proposed in [5] the following two-scale variant of the Cahn-Hilliard equation:

$$
\frac{\partial u}{\partial t}+\varepsilon \Delta^{2} u-\frac{1}{\varepsilon} \Delta f(u)+\lambda_{0} \chi_{\Omega \backslash D}(x)(u-h)=0, \varepsilon>0, \lambda_{0}>0,
$$

in view of applications to binary image inpainting. Here, $h=h(x)$ is a given (damaged) image and $D \subset \Omega$ is the inpainting region ( $\Omega$ is the total region). Furthermore, the term $\lambda_{0} \chi_{\Omega \backslash D}(x)(u-h)$ is the fidelity term ( $\chi$ denotes the indicator function). There are several motivations to use such a term (instead of, e.g., a condition of the form $u=h$ outside the inpainting domain), in particular, in view of the analysis of the model. Indeed, no regularity assumption on $D$ is necessary and no perfect $h$ outside $D$ is required (it could, for instance, be noisy). Finally, the nonlinear term $f$ is regular and cubic, typically, $f(s)=4 s^{3}-6 s^{2}+2 s$. The idea in this model is to solve (1.1) up to steady state in order to obtain an inpainted version $u(x)$ of $h(x)$.

This equation was studied, endowed with Neumann boundary conditions, in [6], [7], [10], [17], and [18].

Well-posedness results for (1.1) have been obtained in [6] (see also [24] for the study of the nonlocal version of the problem and [10] for the study of the stationary problem).

Furthermore, the asymptotic behavior, in terms of finite-dimensional attractors, of (1.1) has been studied in [17]. More precisely, such sets provide information on all the possible dynamics of the system and are expected to have a rich geometric structure; they contain in particular all steady states and heteroclinic orbits. Moreover, the finite-dimensionality means, roughly speaking, that, even though the initial phase space is infinite-dimensional, 
the reduced dynamics can be characterized by a finite number of parameters. We refer the reader to, e.g., [26], [57], and [66] for discussions on this subject.

Finally, the existence of local (in time) solutions to (1.1) with logarithmic nonlinear terms has been studied in [18] (note indeed that the original Cahn-Hilliard equation was actually proposed with thermodynamically relevant logarithmic nonlinear terms which follow from a mean-field model; regular (and, in particular, cubic) nonlinear terms are approximations of such logarithmic nonlinear terms). In that case, we can obtain better results than those obtained with polynomial nonlinear terms in [17], as far as the convergence time is concerned, in particular. We also note that double obstacle nonlinear terms give better results than those obtained with polynomial nonlinear terms, see [7].

Color image inpainting with fourth-order PDE's. We proposed in [19] the following color inpainting model:

$$
\begin{gathered}
\frac{\partial c_{i}}{\partial t}=\Delta \mu_{i}+\lambda_{0} \chi_{\Omega \backslash D}(x)\left(h_{i}-c_{i}\right), \quad i=1, \ldots, n, \\
\mu_{i}=\frac{\partial F(c)}{\partial c_{i}}-\varepsilon^{2} \Delta c_{i}-\frac{1}{n} \sum_{i=1}^{n} \frac{\partial F(c)}{\partial c_{i}}, \quad i=1, \ldots, n,
\end{gathered}
$$

where $F(c)=\frac{1}{n} \sum_{i=1}^{n} c_{i}^{2}\left(1-c_{i}\right)^{2}, h=\left(h_{1}, \ldots, h_{n}\right)$ being the original image and $n$ the number of colors in the original image $h$. System (1.2)-(1.3) is endowed with Neumann boundary conditions. We studied in [19] the well-posedness of the problem, as well as the existence of finite-dimensional attractors. Furthermore, we obtained consistency results with the diphasic model. Finally, we gave numerical simulations which confirm that the one step algorithm with threshold proposed in [17] is efficient, also in the context of multi-color inpainting.

Grayscale image inpainting with fourth-order PDE's. The authors in [10] proposed the following model (total variation in $\mathrm{H}^{-1}$ ):

$$
\frac{\partial u}{\partial t}-\Delta p+\lambda_{0} \chi_{\Omega \backslash D}(x)(u-h)=0, \quad p \in \partial T V(u),
$$

where

$$
T V(u)=\left\{\begin{array}{rr}
|D u| & \text { if }|u(x)| \leq 1 \text { a.e. in } \Omega, \\
+\infty & \text { otherwise }
\end{array}\right.
$$

in view of applications to grayscale image inpainting. In particular, they proved that the corresponding stationary problem has a bounded variation solution.

Furthermore, the authors in [64] proposed two grayscale inpainting models. The first one consists in generalizing the Bertozzi-Esedoglu-Gillette-Cahn-Hilliard model by splitting the grayscale image bit-wise into channels,

$$
u(x) \quad \text { approximated by } \sum_{k=1}^{n} u_{k}(x) 2^{-(k-1)},
$$

where $n>0$ is the number of channels, and then in applying the Bertozzi-EsedogluGillette-Cahn-Hilliard equation to each binary channel $u_{k}$ separately. The second one is 
based on the LCIS (Low Curvature Image Simplifier) model. This latter model reads

$$
\frac{\partial u}{\partial t}+\operatorname{div}(g(\Delta u) \nabla \Delta u)+\lambda_{0} \chi_{\Omega \backslash D}(x)(u-h)=0
$$

where $g$ is the thresholding function, $g(s)=\frac{1}{1+s^{2}}$. Since $g(\Delta u) \nabla \Delta u=\nabla(\arctan (\Delta u))$, we can rewrite the equation as follows:

$$
\frac{\partial u}{\partial t}+\Delta(\arctan (\Delta u))+\lambda_{0} \chi_{\Omega \backslash D}(x)(u-h)=0 .
$$

Note that this model can be seen as a generalization of the Rudin-Osher-Fatemi ([63]) and Perona-Malik ([62]) models.

Finally, the authors in [8] proposed system (1.2)-(1.3) in view of applications to grayscale image inpainting.

Proposed model. Let $u=u_{1}+i u_{2}$ be the phase variable and $\Omega$ be a bounded domain in $\mathbb{R}^{N}(N \leq 3)$ with a regular boundary $\Gamma$. We postulate that the free energy can be written as follows (see also [42]):

$$
\mathcal{E}(u)=\int_{\Omega}\left[\frac{\varepsilon}{2}|-i \nabla u|^{2}+\frac{1}{4 \varepsilon}|u|^{4}-\frac{1}{2 \varepsilon}|u|^{2}\right] d x .
$$

Here and below, we denote by $|\cdot|$ the modulus of a complex number.

Assuming proper boundary conditions, the variation of the energy (1.5) with respect to the phase field $u$ is given by

$$
\partial \mathcal{E}(u)=\int_{\Omega}\left[-\varepsilon \Delta u+\frac{1}{\varepsilon}|u|^{2} u-\frac{1}{\varepsilon} u\right] \partial u d x,
$$

which yields

$$
\frac{\partial \mathcal{E}(u)}{\partial u}=-\varepsilon \Delta u+\frac{1}{\varepsilon}|u|^{2} u-\frac{1}{\varepsilon} u,
$$

and the descent equation can be written as follows:

$$
\frac{\partial u}{\partial t}=-\frac{\partial \mathcal{E}(u)}{\partial u}=\varepsilon \Delta u-\frac{1}{\varepsilon}|u|^{2} u+\frac{1}{\varepsilon} u .
$$

Equation (1.8) can be seen as a complex version of the Ginzburg-Landau (Allen-Cahn) equation. In particular, Grossauer and Scherzer [42] proposed this equation in view of applications to image processing and, more precisely, to image inpainting. In that case, they proposed to treat the image data as complex: the real part of $u(x, t)$ corresponds to the actual grayscale value of the image at $(x, t)$, while the imaginary part is such that $u(x, t)$ is on the boundary of a circle of radius 1 in the complex plane. This leads to a coupled system of equations for $u_{1}(x, t)$ and $u_{2}(x, t)$ which Grossauer and Scherzer solved by an explicit numerical scheme, assuming Dirichlet boundary conditions, $\left.u(x, t)\right|_{\partial D}=$ $\left.u(x, 0)\right|_{\partial D}$. Finally, the authors in [2] proposed a mesh adaptation strategy to solve (1.8) by a finite element scheme.

The complex version of the Cahn-Hilliard equation can be derived exactly as the standard Cahn-Hilliard equation, namely, 
hence

$$
\frac{\partial u}{\partial t}=\Delta \frac{\partial \mathcal{E}}{\partial u}
$$

$$
\frac{\partial u}{\partial t}+\varepsilon \Delta^{2} u-\frac{1}{\varepsilon} \Delta\left(|u|^{2} u-u\right)=0 .
$$

We can note that, as in the case of the complex Ginzburg-Landau equation (1.8), the minima of the nonlinear term satisfy $|u|=1$.

Now, in order for such a model to have applications to image inpainting, we need to add a fidelity term to keep the solution constructed close to the given image in the complement of the inpainting domain, where image information is available. Let $h_{1}$ be the given (damaged) image, $h_{1}: \Omega \rightarrow[-1,1]$. We then introduce the function $h$,

$$
h: \Omega \rightarrow \mathbb{C}, \quad h(x)=h_{1}(x)+i h_{2}(x),
$$

where $h$ satisfies the constraint $|h|=1$, which yields

$$
h_{2}(x)=\sqrt{1-\left(h_{1}(x)\right)^{2}} .
$$

We assume that $h_{1} \in L^{2}(\Omega)$, so that $h_{2} \in L^{2}(\Omega)$ and $h \in L^{2}(\Omega ; \mathbb{C})$; for simplicity, we will also denote by $L^{2}(\Omega)$ the $L^{2}$-functions with values in $\mathbb{C}$ (i.e., $L^{2}(\Omega ; \mathbb{C}$ ); a similar notation will hold for the corresponding Sobolev spaces). We finally consider the following complex Bertozzi-Esedoglu-Gillette-Cahn-Hilliard model:

$$
\frac{\partial u}{\partial t}+\varepsilon \Delta^{2} u-\frac{1}{\varepsilon} \Delta f(u)+\lambda_{0} \chi_{\Omega \backslash D}(x)(u-h)=0,
$$

where $D \Subset \Omega$ is the inpainting domain, $\varepsilon, \lambda_{0}>0$, and $f(z)=|z|^{2} z-z, z \in \mathbb{C}$. This equation is endowed with Neumann boundary conditions as in the original BertozziEsedoglu-Gillette-Cahn-Hilliard equation,

$$
\frac{\partial u}{\partial \nu}=\frac{\partial \Delta u}{\partial \nu}=0, \quad \text { on } \Gamma .
$$

Furthermore, we will assume, in the numerical simulations (but not in the mathematical analysis), that the imaginary part of the initial datum is given by

$$
u_{2}(0, x)=\operatorname{Im}[u(0, x)]=\sqrt{1-(\operatorname{Re}[u(0, x)])^{2}}=\sqrt{1-\left(u_{1}(0, x)\right)^{2}} .
$$

Here and below, $\operatorname{Re}[\varphi]$ and $\operatorname{Im}[\varphi]$ denote the real and the imaginary parts of $\varphi$, respectively.

One main idea when considering Cahn-Hilliard models in image inpainting is to keep the continuity of the isophote vectors at the boundary of the inpainting domain of the constructed image (see [5] and [6]), which is much desirable. Furthermore, compared with other models, the Cahn-Hilliard equation is more efficient, as far as the convergence time is concerned, and allows to reconstruct images with large inpainting regions.

The total variation (in $H^{-1}$ ) model proposed in [10] and the LCIS model proposed in [64] lose some inpainting advantages of the Cahn-Hilliard inpainting. Furthermore, the bitwise model proposed in [64] and the vector-valued model proposed in [8], while preserving the inpainting advantages of the Bertozzi-Esedoglu-Gillette-Cahn-Hilliard model, may be too heavy numerically and may not always be applicable when the number of channels 
(i.e., of shades of gray) in the original damaged image is large, since, in that case, we need to compute the $n$ channels $u_{k}, k=1, \ldots, n$.

Our main aim in this article is to propose a simple model for grayscale image inpainting which preserves the inpainting advantages obtained with the Bertozzi-Esedoglu-GilletteCahn-Hilliard model, i.e., it is not too heavy numerically and is fast, as far as the convergence time is concerned. In particular, we can recover the binary inpainting results obtained in [5] and [6] (see Figure 1) and we only need to compute two solutions (the real and imaginary parts of the order parameter) whatever the number of channels in the damaged image is.

As already encountered in the binary model in [17], one essential difficulty to study the well-posedness and the existence of finite-dimensional attractors is that we no longer have the conservation of mass, i.e., of the spatial average of the order parameter, contrary to the original Cahn-Hilliard equation. A second essential difficulty, when compared to (1.1), is that (1.10) is complex valued (note indeed that (1.1) and (1.10) have exactly the same structure). This makes the derivation of estimates on the nonlinear term (and, consequently, the proof of dissipativity; note that the dissipativity is also used in an essential way in the proof of existence of a solution) much more delicate. This is addressed in Sections 2 to 4. We can also note that the functional framework considered in [19] for the color inpainting model is different from what we have here, since, there, the sum of the components is equal to one.

As far as the numerical simulations are concerned, the authors in [6] give numerical evidence that the steady states of the modified Cahn-Hilliard equation (1.1) are not unique. More precisely, they depend on the initial condition inside the inpainting domain. Furthermore, computing inpainted images with a small $\varepsilon$ only may not allow to extend the level lines into the missing domain as desired. This problem can a priori also appear when solving equation (1.10). Therefore, we follow the strategy devised in [5] and [6], i.e., we consider a dynamic two steps scheme involving the diffuse interface thickness $\varepsilon$. More precisely, we consider a large value of $\varepsilon$ to connect the edges and then a smaller one in order to obtain the inpainting results. Furthermore, when solving (1.10), the solutions may also regularize outside the inpainting domain. Since, in image inpainting, the image is known outside the inpainting region, we use, in the final inpainting results, the information on the image known outside the inpainting domain to obtain better results (see Section 5). In particular, we give numerical simulations with the two steps scheme which preserve the efficiency of the binary inpainting and also confirm that (1.10) gives indeed good results in the context of grayscale inpainting.

Notation. We denote by $\langle u\rangle$ the spatial average of a function $u \in L^{1}(\Omega)$,

$$
\langle u\rangle=\frac{1}{\operatorname{Vol}(\Omega)} \int_{\Omega} u(x) d x,
$$

and set

$$
v=u-\langle u\rangle,
$$

$v$ being the mean-free part of $u$. Furthermore, $\bar{u}$ denotes the conjugate of $u$.

We then set

$$
\dot{H}^{-1}(\Omega)=\left\{\varphi \in H^{-1}(\Omega),\langle\varphi, 1\rangle_{H^{-1}(\Omega), H^{1}(\Omega)}=0\right\}
$$


and

$$
\dot{L}^{2}(\Omega)=\left\{\varphi \in L^{2}(\Omega),\langle\varphi\rangle=0\right\} .
$$

We denote by $((\cdot, \cdot))$ the usual $L^{2}$-Hermitian product, with associated norm $\|\cdot\|$; in particular, for two complex valued functions $\varphi$ and $\psi$ in $L^{2}(\Omega)$,

$$
((\varphi, \psi))=\int_{\Omega} \varphi \bar{\psi} d x
$$

We further set $\|\cdot\|_{-1}=\left\|(-\Delta)^{-1} \cdot\right\|$, where $(-\Delta)^{-1}$ denotes the inverse minus Laplace operator associated with Neumann boundary conditions and acting on functions with null spatial average. More generally, $\|\cdot\|_{X}$ denotes the norm on the Banach space $X$.

Throughout the article, the same letters $c$ and $c^{\prime}$ denote (generally positive) constants which may vary from line to line, or even in a same line. Similarly, the same letter $Q$ denotes monotone increasing (with respect to each argument) functions which may vary from line to line, or even in a same line. In general, these quantities depend on $\varepsilon$ and $\lambda_{0}$.

\section{A PRIORI ESTIMATES}

We assume in Sections 2 to 4 that $h \in L^{2}(\Omega)$; in particular, $h$ does not necessarily satisfy the constraint $|h|=1$.

Estimates on the nonlinear term. We first have the

Proposition 2.1. Let $u \in L^{4}(\Omega)$. Then, there exists $c_{0}>0$ such that

$$
\boldsymbol{\operatorname { R e }}[((f(v+\langle u\rangle)-f(\langle u\rangle), v))] \geq c_{0} \int_{\Omega}\left(|v|^{4}+|v|^{2}|\langle u\rangle|^{2}+(\boldsymbol{\operatorname { R e }}[\bar{v}\langle u\rangle])^{2}\right) d x-\|v\|^{2} .
$$

Proof. We first have

$$
\begin{aligned}
f(v+\langle u\rangle)-f(\langle u\rangle)= & \left(|v|^{2}+|\langle u\rangle|^{2}+2 \mathbf{R e}[v] \mathbf{R e}[\langle u\rangle]+2 \operatorname{Im}[v] \mathbf{I m}[\langle u\rangle]-1\right) v \\
& +\left(|v|^{2}+2 \operatorname{Re}[v] \mathbf{R e}[\langle u\rangle]+2 \mathbf{I m}[v] \mathbf{I m}[\langle u\rangle]\right)\langle u\rangle .
\end{aligned}
$$

Noting that

$$
\boldsymbol{\operatorname { R e }}[\bar{v}\langle u\rangle]=\operatorname{Re}[v] \boldsymbol{\operatorname { R e }}[\langle u\rangle]+\operatorname{Im}[v] \operatorname{Im}[\langle u\rangle]
$$

it follows that

$$
\begin{aligned}
{[f(v+\langle u\rangle)-f(\langle u\rangle)] \bar{v}=} & \left(|v|^{2}+|\langle u\rangle|^{2}+2 \operatorname{Re}[\bar{v}\langle u\rangle]-1\right)|v|^{2} \\
& +\left(|v|^{2}+2 \operatorname{Re}[\bar{v}\langle u\rangle]\right)\langle u\rangle \bar{v}
\end{aligned}
$$

and

$$
\begin{aligned}
\boldsymbol{\operatorname { R e }}[(f(v+\langle u\rangle)-f(\langle u\rangle)) \bar{v}]= & \left(|v|^{2}+|\langle u\rangle|^{2}+2 \operatorname{Re}[\bar{v}\langle u\rangle]-1\right)|v|^{2} \\
& +\left(|v|^{2}+2 \operatorname{Re}[\bar{v}\langle u\rangle]\right) \operatorname{Re}[\bar{v}\langle u\rangle]
\end{aligned}
$$


Integrating over $\Omega$, we obtain

$$
\begin{aligned}
\boldsymbol{\operatorname { R e }}[((f(v+\langle u\rangle)-f(\langle u\rangle), v))] \\
\quad=\int_{\Omega}\left[|v|^{4}+|v|^{2}|\langle u\rangle|^{2}+2(\boldsymbol{R e}[\bar{v}\langle u\rangle])^{2}\right] d x+3 \int_{\Omega} \boldsymbol{\operatorname { R e }}[\bar{v}\langle u\rangle]|v|^{2} d x-\|v\|^{2} \\
\quad \geq \int_{\Omega}\left[|v|^{4}+|v|^{2}|\langle u\rangle|^{2}+2(\boldsymbol{R e}[\bar{v}\langle u\rangle])^{2}\right] d x-3 \int_{\Omega}|\boldsymbol{R e}[\bar{v}\langle u\rangle]||v|^{2} d x-\|v\|^{2} .
\end{aligned}
$$

Using properties of complex numbers, namely,

$$
|\boldsymbol{R e}[z]| \leq|z| \quad \text { and } \quad\left|z z^{\prime}\right|=|z|\left|z^{\prime}\right|, \quad \forall z, z^{\prime} \in \mathbb{C}
$$

we find

$$
\begin{aligned}
& 3 \int_{\Omega}|\boldsymbol{R e}[\bar{v}\langle u\rangle]||v|^{2} d x=2 \int_{\Omega}|\boldsymbol{R e}[\bar{v}\langle u\rangle]||v|^{2} d x+\int_{\Omega}|\boldsymbol{R e}[\bar{v}\langle u\rangle]||v|^{2} d x \\
& \leq \alpha \int_{\Omega}|v|^{4} d x+\beta \int_{\Omega}|\boldsymbol{R e}[\bar{v}\langle u\rangle]|^{2} d x+\gamma \int_{\Omega}|v|^{4} d x+\zeta \int_{\Omega}|v|^{2}|\langle u\rangle|^{2} d x
\end{aligned}
$$

where $\alpha, \beta, \gamma, \zeta$ are nonnegative constants which satisfy $\alpha+\gamma<1, \beta<2$, and $\zeta<1$. The last inequality has been obtained by employing Young's inequality (e.g., $\alpha=\frac{21}{41}, \beta=$ $\frac{41}{21}, \gamma=\frac{3}{8}$, and $\zeta=\frac{2}{3}$ ). We finally deduce that

$$
\boldsymbol{\operatorname { R e }}[((f(v+\langle u\rangle)-f(\langle u\rangle), v))] \geq c_{0} \int_{\Omega}\left(|v|^{4}+|v|^{2}|\langle u\rangle|^{2}+(\boldsymbol{\operatorname { R e }}[\bar{v}\langle u\rangle])^{2}\right) d x-\|v\|^{2}
$$

where $c_{0}>0$.

Proposition 2.2. Let $w \in H^{1}(\Omega)$. Then,

$$
\operatorname{Re}[((\nabla f(w), \nabla w))] \geq-\|\nabla w\|^{2}
$$

Proof. We first note that

$$
((\nabla f(w), \nabla w))=\int_{\Omega}|w|^{2}|\nabla w|^{2} d x+2 \sum_{j=1}^{N} \int_{\Omega} \operatorname{Re}\left[w \frac{\partial \bar{w}}{\partial x_{j}}\right]\left(w \frac{\partial \bar{w}}{\partial x_{j}}\right) d x-\|\nabla w\|^{2},
$$

hence

$$
\begin{aligned}
\operatorname{Re}[((\nabla f(w), \nabla w))] & =\int_{\Omega}|w|^{2}|\nabla w|^{2} d x+2 \sum_{j=1}^{N} \int_{\Omega}\left(\operatorname{Re}\left[w \frac{\partial \bar{w}}{\partial x_{j}}\right]\right)^{2} d x-\|\nabla w\|^{2} \\
& \geq-\|\nabla w\|^{2}
\end{aligned}
$$


Absorbing set in $\dot{H}^{-1}(\Omega)$. We first recall that $\mathcal{B}_{0}$ is an absorbing set if it is bounded and if, for every bounded subset $\mathcal{B}, \exists t_{0}=t_{0}(\mathcal{B})$ such that $u_{0} \in \mathcal{B}$ and $t \geq t_{0} \Longrightarrow$ $u(t) \in \mathcal{B}_{0}, u_{0}$ being the initial datum. When such a set exists, the associated dynamical system is called dissipative.

Integrating (1.10) over $\Omega$, we have

$$
\frac{d}{d t}\langle u\rangle+\lambda_{0}\left\langle\chi_{\Omega \backslash D}(x)(u-h)\right\rangle=0
$$

We thus rewrite (1.10) as follows:

$$
\frac{\partial v}{\partial t}+\varepsilon \Delta^{2} v-\frac{1}{\varepsilon} \Delta f(u)+\lambda_{0} \chi_{\Omega \backslash D}(x)(u-h)-\lambda_{0}\left\langle\chi_{\Omega \backslash D}(x)(u-h)\right\rangle=0
$$

and (2.2) is equivalent to

$$
\begin{gathered}
\frac{\partial(-\Delta)^{-1} v}{\partial t}-\varepsilon \Delta v+\frac{1}{\varepsilon}(f(v+\langle u\rangle)-\langle f(u)\rangle) \\
+\lambda_{0}(-\Delta)^{-1}\left(\chi_{\Omega \backslash D}(x)(u-h)-\left\langle\chi_{\Omega \backslash D}(x)(u-h)\right\rangle\right)=0 .
\end{gathered}
$$

We further have the

Lemma 2.3. For every $u \in L^{4}(\Omega)$, there holds

$$
\begin{gathered}
\lambda_{0}\left|\boldsymbol{\operatorname { R e }}\left[\left(\left((-\Delta)^{-1}\left(\chi_{\Omega \backslash D}(x)(u-h)-\left\langle\chi_{\Omega \backslash D}(x)(u-h)\right\rangle\right), v\right)\right)\right]\right| \\
\quad \leq \frac{c_{0}}{2 \varepsilon} \int_{\Omega}\left(|v|^{4}+|v|^{2}|\langle u\rangle|^{2}+(\boldsymbol{\operatorname { R e }}[\bar{v}\langle u\rangle])^{2}\right) d x+c\|h\|^{2}+c^{\prime} .
\end{gathered}
$$

Proof. We first note that

$$
\begin{gathered}
\lambda_{0}\left|\boldsymbol{\operatorname { R e }}\left[\left(\left((-\Delta)^{-1}\left(\chi_{\Omega \backslash D}(x)(u-h)-\left\langle\chi_{\Omega \backslash D}(x)(u-h)\right\rangle\right), v\right)\right)\right]\right| \\
\quad \leq \lambda_{0}\left|\left(\left((-\Delta)^{-1}\left(\chi_{\Omega \backslash D}(x)(u-h)-\left\langle\chi_{\Omega \backslash D}(x)(u-h)\right\rangle\right), v\right)\right)\right| .
\end{gathered}
$$

Since $\langle v\rangle=0$, we have

$$
\begin{array}{r}
\lambda_{0}\left|\left(\left((-\Delta)^{-1}\left(\chi_{\Omega \backslash D}(x)(u-h)-\left\langle\chi_{\Omega \backslash D}(x)(u-h)\right\rangle\right), v\right)\right)\right| \\
=\lambda_{0}\left|\left(\left(\chi_{\Omega \backslash D}(x)(u-h),(-\Delta)^{-1} v\right)\right)\right|
\end{array}
$$


and Hölder's inequality yields

$$
\begin{aligned}
& \lambda_{0}\left|\left(\left(\chi_{\Omega \backslash D}(x)(u-h),(-\Delta)^{-1} v\right)\right)\right| \\
& \quad \leq c\|u-h\|\|v\| \\
& \quad \leq c\left(\|v\|^{2}+|\langle u\rangle|\|v\|\right)+c^{\prime}\|h\|^{2} \\
& \quad \leq \frac{c_{0}}{2 \varepsilon} \int_{\Omega}\left(|v|^{4}+|v|^{2}|\langle u\rangle|^{2}+(\operatorname{Re}[\bar{v}\langle u\rangle])^{2}\right) d x+c\|h\|^{2}+c^{\prime}
\end{aligned}
$$

Proposition 2.4. Problem (1.10) has an absorbing set in $\dot{H}^{-1}(\Omega)$.

Proof. We multiply (2.3) by $\bar{v}$ and have, integrating over $\Omega$ and by parts and taking the real part,

$$
\begin{aligned}
\frac{1}{2} \frac{d}{d t}\|v\|_{-1}^{2}+\varepsilon\|\nabla v\|^{2}+\frac{1}{\varepsilon} \operatorname{Re}[((f(v+\langle u\rangle)-f(\langle u\rangle), v))] \\
+\lambda_{0} \operatorname{Re}\left[\left(\left((-\Delta)^{-1}\left(\chi_{\Omega \backslash D}(x)(u-h)-\left\langle\chi_{\Omega \backslash D}(x)(u-h)\right\rangle\right), v\right)\right)\right]=0 .
\end{aligned}
$$

It follows from Proposition 2.1 and Lemma 2.3 that

$$
\begin{gathered}
\frac{1}{2} \frac{d}{d t}\|v\|_{-1}^{2}+\varepsilon\|\nabla v\|^{2}+\frac{c_{0}}{2 \varepsilon} \int_{\Omega} \\
\left(|v|^{4}+|v|^{2}|\langle u\rangle|^{2}+(\boldsymbol{R e}[\bar{v}\langle u\rangle])^{2}\right) d x \\
\leq c\left(\|v\|^{2}+\|h\|^{2}+1\right)
\end{gathered}
$$

hence

$$
\frac{d}{d t}\|v\|_{-1}^{2}+\varepsilon\|\nabla v\|^{2}+\frac{c_{0}}{\varepsilon} \int_{\Omega}\left(|v|^{4}+|v|^{2}|\langle u\rangle|^{2}+(\boldsymbol{\operatorname { R e }}[\bar{v}\langle u\rangle])^{2}\right) d x \leq c .
$$

In particular, it follows from (2.6) that

$$
\frac{d}{d t}\|v\|_{-1}^{2}+c\|v\|_{-1}^{2} \leq c^{\prime}, \quad c>0
$$

We finally deduce from Gronwall's lemma that

$$
\|v\|_{-1}^{2} \leq e^{-c t}\left\|v_{0}\right\|_{-1}^{2}+c^{\prime}, \quad c>0, \forall t \geq 0
$$

where the constants $c$ and $c^{\prime}$ are independent of $v_{0}$ and $t$, hence the result.

Absorbing set in $\dot{L}^{2}(\Omega)$. As a consequence of (2.6) and (2.8), we have the

Corollary 2.5. We assume that $v_{0}$ belongs to a bounded subset $\mathcal{B}$ of $\dot{H}^{-1}(\Omega)$. Then, there exists $t_{0}=t_{0}(\mathcal{B}) \geq 0$ such that, for every $t \geq t_{0}$, there holds

$$
\int_{t}^{t+r}\|\nabla v\|^{2} d s \leq c(r)
$$

and

$$
\int_{t}^{t+r} \int_{\Omega}\left(|v|^{4}+|v|^{2}|\langle u\rangle|^{2}+(\boldsymbol{R e}[\bar{v}\langle u\rangle])^{2}\right) d x d s \leq c(r),
$$


$r>0$ given.

We further have the

Lemma 2.6. For every $u \in L^{4}(\Omega)$, there holds

$$
\begin{array}{r}
\lambda_{0}\left|\boldsymbol{\operatorname { R e }}\left[\left(\left(\chi_{\Omega \backslash D}(x)(u-h)-\left\langle\chi_{\Omega \backslash D}(x)(u-h)\right\rangle, v\right)\right)\right]\right| \\
\leq \frac{c_{0}}{2 \varepsilon} \int_{\Omega}\left(|v|^{4}+|v|^{2}|\langle u\rangle|^{2}+(\boldsymbol{\operatorname { R e }}[\bar{v}\langle u\rangle])^{2}\right) d x+c\|h\|^{2}+c^{\prime} .
\end{array}
$$

Remark 2.7. The proof of Lemma 2.6 is similar to the one of Lemma 2.3.

Proposition 2.8. Problem (1.10) has an absorbing set in $\dot{L}^{2}(\Omega)$.

Proof. We multiply (2.2) by $\bar{v}$ and have

$$
\begin{aligned}
\frac{1}{2} \frac{d}{d t}\|v\|^{2}+\varepsilon\|\Delta v\|^{2}+\frac{1}{\varepsilon} \operatorname{Re}[((\nabla f(u), \nabla u))] \\
+\lambda_{0} \operatorname{Re}\left[\left(\left(\chi_{\Omega \backslash D}(x)(u-h)-\left\langle\chi_{\Omega \backslash D}(x)(u-h)\right\rangle, v\right)\right)\right]=0 .
\end{aligned}
$$

It follows from Proposition 2.2 and Lemma 2.6 that

$$
\begin{aligned}
& \frac{1}{2} \frac{d}{d t}\|v\|^{2}+\varepsilon\|\Delta v\|^{2} \leq \frac{1}{\varepsilon}\|\nabla v\|^{2}+c\|h\|^{2}+c^{\prime} \\
+ & \frac{c_{0}}{2 \varepsilon} \int_{\Omega}\left(|v|^{4}+|v|^{2}|\langle u\rangle|^{2}+(\operatorname{Re}[\bar{v}\langle u\rangle])^{2}\right) d x .
\end{aligned}
$$

We then deduce from Corollary 2.5 and the uniform Gronwall's lemma that

$$
\|v\|^{2} \leq c, \quad \forall t \geq t_{0}+r
$$

where the constant $c$ is independent of $v_{0}$ and $t$. Finally, integrating (2.6) and (2.10) between 0 and $t_{0}+r$ and using (2.11), we obtain

$$
\|v\|^{2} \leq Q\left(\left\|v_{0}\right\|\right), \quad \forall t \geq 0
$$

Dissipativity of the spatial average. We have the

Proposition 2.9. Let $u$ be a solution to (1.10). Then, for every $u_{0} \in L^{2}(\Omega)$, the spatial average of the order parameter satisfies

$$
|\langle u\rangle| \leq\left(Q\left(\left\|v_{0}\right\|\right)+\left|\left\langle u_{0}\right\rangle\right|\right) e^{-c t}+c^{\prime}, \quad c>0, \forall t \geq 0,
$$

where $c=c\left(\varepsilon, \lambda_{0}\right)$ and $c^{\prime}=c^{\prime}\left(\varepsilon, \lambda_{0}\right)$ are two constants which are independent of $u_{0}$ and $t$. Proof. Setting $u=\langle u\rangle+v$ in (2.1), we have

$$
\frac{d}{d t}\langle u\rangle+\frac{\lambda_{0}}{\operatorname{Vol}(\Omega)} \int_{\Omega \backslash D}(\langle u\rangle+v-h) d x=0 .
$$

Therefore,

$$
\frac{d}{d t}\langle u\rangle+\xi\langle u\rangle=-\frac{\lambda_{0}}{\operatorname{Vol}(\Omega)} \int_{\Omega \backslash D}(v-h) d x,
$$


where $\xi=\frac{\lambda_{0} \operatorname{Vol}(\Omega \backslash D)}{\operatorname{Vol}(\Omega)}$, hence

$$
\frac{d}{d t}\left(e^{\xi t}\langle u\rangle\right)=-\frac{\lambda_{0}}{\operatorname{Vol}(\Omega)} e^{\xi t} \int_{\Omega \backslash D}(v-h) d x
$$

and

$$
\langle u\rangle=e^{-\xi t}\left\langle u_{0}\right\rangle-\frac{\lambda_{0}}{\operatorname{Vol}(\Omega)} e^{-\xi t} \int_{0}^{t} e^{\xi s} \int_{\Omega \backslash D}(v-h) d x d s .
$$

Noting that

$$
\left|z+z^{\prime}\right| \leq|z|+\left|z^{\prime}\right|, \quad \forall z, z^{\prime} \in \mathbb{C},
$$

we find

$$
|\langle u\rangle| \leq e^{-\xi t}\left|\left\langle u_{0}\right\rangle\right|+c e^{-\xi t} \int_{0}^{t} e^{\xi s}(\|v\|+\|h\|) d s, \quad \forall t \geq 0,
$$

where $c=\frac{\lambda_{0}}{\operatorname{Vol}(\Omega)^{\frac{1}{2}}}$. We then deduce from (2.11) and (2.12) that

$$
|\langle u\rangle| \leq\left(Q\left(\left\|v_{0}\right\|\right)+\left|\left\langle u_{0}\right\rangle\right|\right) e^{-c t}+c^{\prime}, \quad c>0, \forall t \geq 0,
$$

where the constants $c$ and $c^{\prime}$ are independent of $u_{0}$ and $t$, hence the result.

Absorbing set in $H^{2}(\Omega)$. We have the

Lemma 2.10. Let $u$ be a regular solution to (1.10). Then,

$$
\left|\boldsymbol{R e}\left[\left(\left(\Delta f(u), \Delta^{2} u\right)\right)\right]\right| \leq \frac{\varepsilon^{2}}{4}\left\|\Delta^{2} u\right\|^{2}+c
$$

and

$$
\left|\boldsymbol{\operatorname { R e }}\left[\left(\left(\chi_{\Omega \backslash D}(x)(u-h), \Delta^{2} u\right)\right)\right]\right| \leq \frac{\varepsilon}{4 \lambda_{0}}\left\|\Delta^{2} u\right\|^{2}+c\left(\|u\|^{2}+\|h\|^{2}\right) .
$$

Proof. First, observe that

$$
\begin{aligned}
\left|\boldsymbol{\operatorname { R e }}\left[\left(\left(\Delta f(u), \Delta^{2} u\right)\right)\right]\right| & \leq\left|\left(\left(\Delta f(u), \Delta^{2} u\right)\right)\right| \\
& \leq\|\Delta f(u)\|\left\|\Delta^{2} u\right\| \\
& \leq \frac{2}{\varepsilon^{2}}\|\Delta f(u)\|^{2}+\frac{\varepsilon^{2}}{8}\left\|\Delta^{2} u\right\|^{2} .
\end{aligned}
$$

We further have (see, e.g., [66])

$$
\|\Delta f(u)\|^{2} \leq \frac{\varepsilon^{4}}{16}\left\|\Delta^{2} u\right\|^{2}+c
$$

and, owing to Young's inequality,

$$
\left|\boldsymbol{\operatorname { R e }}\left[\left(\left(\Delta f(u), \Delta^{2} u\right)\right)\right]\right| \leq \frac{\varepsilon^{2}}{4}\left\|\Delta^{2} u\right\|^{2}+c .
$$


On the other hand, we have

$$
\begin{aligned}
\left|\boldsymbol{\operatorname { R e }}\left[\left(\left(\chi_{\Omega \backslash D}(x)(u-h), \Delta^{2} u\right)\right)\right]\right| & \leq\left|\left(\left(\chi_{\Omega \backslash D}(x)(u-h), \Delta^{2} u\right)\right)\right| \\
& \leq\|u-h\|\left\|\Delta^{2} u\right\| \\
& \leq \frac{\varepsilon}{4 \lambda_{0}}\left\|\Delta^{2} u\right\|^{2}+c\left(\|u\|^{2}+\|h\|^{2}\right) .
\end{aligned}
$$

Lemma 2.11. Let $u$ be a solution to (1.10). Then,

$$
\frac{d}{d t}|\langle u\rangle|^{2} \leq c\left(\|v\|^{2}+|\langle u\rangle|^{2}+\|h\|^{2}\right) .
$$

Proof. We first have

$$
\frac{d}{d t}|\langle u\rangle|^{2}=\frac{d}{d t}(\boldsymbol{R e}[\langle u\rangle])^{2}+\frac{d}{d t}(\operatorname{Im}[\langle u\rangle])^{2} .
$$

Noting that, owing to (2.1),

$$
\frac{d}{d t} \boldsymbol{R e}[\langle u\rangle]=-\lambda_{0}\left\langle\chi_{\Omega \backslash D}(x) \mathbf{R e}[u-h]\right\rangle
$$

and

it follows that

$$
\frac{d}{d t} \operatorname{Im}[\langle u\rangle]=-\lambda_{0}\left\langle\chi_{\Omega \backslash D}(x) \operatorname{Im}[u-h]\right\rangle
$$

$$
\begin{aligned}
\frac{d}{d t}(\boldsymbol{\operatorname { R e }}[\langle u\rangle])^{2} & =2 \boldsymbol{R e}[\langle u\rangle] \frac{d}{d t} \boldsymbol{\operatorname { R e }}[\langle u\rangle] \\
& \leq c|\langle u\rangle|\left|\int_{\Omega \backslash D} \boldsymbol{\operatorname { R e }}[u-h] d x\right| \\
& \leq c\left(\|v\|^{2}+|\langle u\rangle|^{2}+\|h\|^{2}\right)
\end{aligned}
$$

and, similarly,

$$
\frac{d}{d t}(\operatorname{Im}[\langle u\rangle])^{2} \leq c\left(\|v\|^{2}+|\langle u\rangle|^{2}+\|h\|^{2}\right)
$$

We finally deduce that

$$
\frac{d}{d t}|\langle u\rangle|^{2} \leq c\left(\|v\|^{2}+|\langle u\rangle|^{2}+\|h\|^{2}\right)
$$

Proposition 2.12. Problem (1.10) has an absorbing set in $H^{2}(\Omega)$ which is compact in $L^{2}(\Omega)$.

Proof. We multiply (1.10) by $\Delta^{2} \bar{u}$ and have

$$
\frac{1}{2} \frac{d}{d t}\|\Delta u\|^{2}+\varepsilon\left\|\Delta^{2} u\right\|^{2}+\frac{1}{\varepsilon} \boldsymbol{R e}\left[\left(\left(\Delta f(u), \Delta^{2} u\right)\right)\right]+\lambda_{0} \mathbf{R e}\left[\left(\left(\chi_{\Omega \backslash D}(x)(u-h), \Delta^{2} u\right)\right)\right]=0
$$
hence, owing to Lemma 2.10,

$$
\frac{1}{2} \frac{d}{d t}\|\Delta u\|^{2}+\frac{\varepsilon}{2}\left\|\Delta^{2} u\right\|^{2} \leq c\left(\|v\|^{2}+|\langle u\rangle|^{2}+\|h\|^{2}\right) .
$$


It then follows from Lemma 2.11 that

$$
\frac{d}{d t}\left(\|\Delta u\|^{2}+|\langle u\rangle|^{2}\right)+\varepsilon\left\|\Delta^{2} u\right\|^{2} \leq c\left(\|v\|^{2}+|\langle u\rangle|^{2}+\|h\|^{2}\right), \quad \forall t \geq 0 .
$$

Assuming that $u_{0}$ belongs to a bounded subset $\mathcal{B}$ of $L^{2}(\Omega)$, we further note that Proposition 2.8 and Proposition 2.9 yield that there exists $t_{1}=t_{1}(\mathcal{B}) \geq 0$ such that

$$
\|v\|^{2} \leq c \quad \text { and } \quad|\langle u\rangle|^{2} \leq c, \quad \forall t \geq t_{1},
$$

where the constant $c$ is independent of $u_{0}$ and $t$. We also have, integrating (2.10) over $(t, t+r)$, for $r \in(0,1)$ fixed, and owing to $(2.11)$,

$$
\int_{t}^{t+r}\|\Delta u\|^{2} d s \leq c(r) .
$$

We finally deduce from $(2.15),(2.16),(2.17)$, and the uniform Gronwall's lemma that

$$
\|u\|_{H^{2}(\Omega)}^{2} \leq c, \quad \forall t \geq t_{1}+r, r>0 \text { fixed },
$$

where the constant $c$ is independent of $u_{0}$ and $t$.

\section{Well-POSEDNESS AND EXISTENCE of THE GLOBAL ATtRACTOR}

\section{a) Well-posedness.}

Proposition 3.1. Let $z$ and $z^{\prime}$ be regular solutions to (1.10). Then, there exists a nonnegative constant $c_{0}>0$ such that

$\boldsymbol{\operatorname { R e }}\left[\left(\left(f(z)-f\left(z^{\prime}\right), z-z^{\prime}\right)\right)\right] \geq c_{0} \int_{\Omega}\left(\left|z-z^{\prime}\right|^{4}+|z|^{2}\left|z-z^{\prime}\right|^{2}+\left(\boldsymbol{\operatorname { R e }}\left[z\left(\overline{z-z^{\prime}}\right)\right]\right)^{2}\right) d x-\left\|z-z^{\prime}\right\|^{2}$

and

$$
\begin{aligned}
& \left|\boldsymbol{\operatorname { R e }}\left[\left(\left(f(z)-f\left(z^{\prime}\right),\left\langle z-z^{\prime}\right\rangle\right)\right)\right]\right| \\
& \leq c\left(\|z\|_{L^{4}(\Omega)}^{2}+\left\|z^{\prime}\right\|_{L^{4}(\Omega)}^{2}+1\right)\left(\left\|z-z^{\prime}\right\|^{2}+\left|\left\langle z-z^{\prime}\right\rangle\right|^{2}\right) .
\end{aligned}
$$

Proof. Setting $w=z-z^{\prime}$, we have

$$
\left(\left(f(z)-f\left(z^{\prime}\right), z-z^{\prime}\right)\right)=\left(\left(|z|^{2} z-|z-w|^{2}(z-w)-w, w\right)\right)
$$

and, using the fact that

$$
|z-w|^{2}=|z|^{2}+|w|^{2}-2 \operatorname{Re}[z \bar{w}]
$$

we find

$$
\left(\left(f(z)-f\left(z^{\prime}\right), z-z^{\prime}\right)\right)=\left(\left(|w|^{2} w-|w|^{2} z+|z|^{2} w+2 \operatorname{Re}[z \bar{w}] z-2 \operatorname{Re}[z \bar{w}] w-w, w\right)\right) .
$$

It thus follows that

$$
\begin{gathered}
\operatorname{Re}\left[\left(\left(f(z)-f\left(z^{\prime}\right), z-z^{\prime}\right)\right)\right]= \\
\int_{\Omega}\left(|w|^{4}+|z|^{2}|w|^{2}+2(\boldsymbol{\operatorname { R e }}[z \bar{w}])^{2}\right) d x-3 \int_{\Omega}|w|^{2} \mathbf{R e}[z \bar{w}] d x-\int_{\Omega}|w|^{2} d x
\end{gathered}
$$


which yields, owing to Young's inequality (see the proof of Proposition 2.1),

$$
\boldsymbol{\operatorname { R e }}\left[\left(\left(f(z)-f\left(z^{\prime}\right), z-z^{\prime}\right)\right)\right] \geq c_{0} \int_{\Omega}\left(|w|^{4}+|z|^{2}|w|^{2}+(\boldsymbol{\operatorname { R e }}[z \bar{w}])^{2}\right) d x-\int_{\Omega}|w|^{2} d x
$$

where $c_{0}>0$.

On the other hand, we have

$$
\begin{gathered}
\boldsymbol{\operatorname { R e }}\left[\left(\left(f(z)-f\left(z^{\prime}\right),\left\langle z-z^{\prime}\right\rangle\right)\right)\right]=\operatorname{Re}\left[\left\langle z-z^{\prime}\right\rangle\right] \int_{\Omega} \boldsymbol{\operatorname { R e }}\left[f(z)-f\left(z^{\prime}\right)\right] d x \\
+\operatorname{Im}\left[\left\langle z-z^{\prime}\right\rangle\right] \int_{\Omega} \mathbf{I m}\left[f(z)-f\left(z^{\prime}\right)\right] d x .
\end{gathered}
$$

Observe now that

$$
\left|f^{\prime}(z)\right| \leq 3|z|^{2}+1
$$

Therefore,

$$
\begin{gathered}
\left|\boldsymbol{\operatorname { R e }}\left[\left\langle z-z^{\prime}\right\rangle\right] \int_{\Omega} \boldsymbol{\operatorname { R e }}\left[f(z)-f\left(z^{\prime}\right)\right] d x\right| \\
=\left|\boldsymbol{\operatorname { R e }}\left[\left\langle z-z^{\prime}\right\rangle\right] \int_{\Omega} \boldsymbol{\operatorname { R e }}[w] \int_{0}^{1} \boldsymbol{\operatorname { R e }}\left[f^{\prime}\left(z+s\left(z-z^{\prime}\right)\right)\right] d s d x\right| \\
\leq c|\langle w\rangle| \int_{\Omega}\left(|z|^{2}+\left|z^{\prime}\right|^{2}+1\right)|w| d x \\
\leq c\left(\|z\|_{L^{4}(\Omega)}^{2}+\left\|z^{\prime}\right\|_{L^{4}(\Omega)}^{2}+1\right)\left(\|w\|^{2}+|\langle w\rangle|^{2}\right) .
\end{gathered}
$$

Similarly,

$$
\begin{gathered}
\left|\operatorname{Im}\left[\left\langle z-z^{\prime}\right\rangle\right] \int_{\Omega} \operatorname{Im}\left[f(z)-f\left(z^{\prime}\right)\right] d x\right| \\
\leq c\left(\|z\|_{L^{4}(\Omega)}^{2}+\left\|z^{\prime}\right\|_{L^{4}(\Omega)}^{2}+1\right)\left(\|w\|^{2}+|\langle w\rangle|^{2}\right),
\end{gathered}
$$

so that

$$
\begin{gathered}
\left|\boldsymbol{\operatorname { R e }}\left[\left(\left(f(z)-f\left(z^{\prime}\right),\left\langle z-z^{\prime}\right\rangle\right)\right)\right]\right| \\
\leq c\left(\|z\|_{L^{4}(\Omega)}^{2}+\left\|z^{\prime}\right\|_{L^{4}(\Omega)}^{2}+1\right)\left(\|w\|^{2}+|\langle w\rangle|^{2}\right) .
\end{gathered}
$$

Theorem 3.2. For every $u_{0} \in L^{2}(\Omega)$ and every $T>0$, the initial-boundary value problem associated with (1.10) has a unique solution $u$ such that

$$
u \in L^{\infty}\left([0, T], L^{2}(\Omega)\right) \cap L^{2}\left([0, T], H^{2}(\Omega)\right) \cap L^{4}\left([0, T], L^{4}(\Omega)\right) .
$$


Proof. We first note that it is easy to show that the operator $(-\Delta)^{-1}$ is self-adjoint and compact in $\dot{L}^{2}(\Omega)$. There thus exists an orthonormal basis of $\dot{L}^{2}(\Omega)$ associated with the eigenvalues $\lambda_{j}, j \geq 1$, of $-\Delta$,

$$
\left\{\begin{aligned}
\lambda_{1} & =\inf _{\varphi \in\left(H^{1}(\Omega) \cap \dot{L}^{2}(\Omega)\right) \backslash\{0\}} \frac{\|\varphi\|_{H^{1}(\Omega)}^{2}}{\|\varphi\|^{2}}, \\
-\Delta N_{j} & =\lambda_{j} N_{j}, \quad N_{j} \in H^{2}(\Omega) \cap \dot{L}^{2}(\Omega), \quad \frac{\partial N_{j}}{\partial \nu}=0, \text { on } \Gamma, \quad j \geq 1, \\
0 & <\lambda_{1} \leq \lambda_{2} \leq \ldots,
\end{aligned}\right.
$$

where the family $\left\{N_{j}\right\}_{j}$ is assumed to be normalized in the norm of $\dot{L}^{2}(\Omega)$, i.e.,

$$
\left(\left(N_{i}, N_{j}\right)\right)=\delta_{i j}
$$

where

$$
\delta_{i j}= \begin{cases}1 & \text { if } i=j \\ 0 & \text { otherwise }\end{cases}
$$

We denote by $E_{m}$ the space

$$
E_{m}=\operatorname{span}\left\{N_{1}, N_{2}, \ldots, N_{m}\right\}
$$

and by $P_{m}$ the orthogonal projection from $H^{1}(\Omega) \cap \dot{L}^{2}(\Omega)$ onto $E_{m}$,

$$
P_{m} h=\sum_{j=1}^{m}\left(\left(h, N_{j}\right)\right) N_{j} .
$$

The variational formulation of the problem reads: Find $u_{m}:[0, T] \rightarrow\left\langle u_{m}\right\rangle+E_{m}$, $u_{m}(t)=\left\langle u_{m}(t)\right\rangle+v_{m}(t)=\left\langle u_{m}(t)\right\rangle+\sum_{j=1}^{m} u_{m_{j}}(t) N_{j}$, such that

$$
\begin{aligned}
& \frac{d}{d t}\left(\left((-\Delta)^{-1} \sum_{i=1}^{m} u_{m_{i}}(t) N_{i}, N_{j}\right)\right) \\
& \quad+\varepsilon\left(\left(\sum_{i=1}^{m} u_{m_{i}}(t) \nabla N_{i}, \nabla N_{j}\right)\right)+\frac{1}{\varepsilon}\left(\left(f\left(\left\langle u_{m}\right\rangle+v_{m}\right), N_{j}\right)\right) \\
& \quad-\frac{1}{\varepsilon}\left(\left(\left\langle f\left(\left\langle u_{m}\right\rangle+v_{m}\right)\right\rangle, N_{j}\right)\right)+\lambda_{0}\left(\left((-\Delta)^{-1}\left(\chi_{\Omega \backslash D}(x)\left(\left\langle u_{m}\right\rangle+v_{m}-h\right)\right), N_{j}\right)\right) \\
& -\lambda_{0}\left(\left((-\Delta)^{-1}\left(\left\langle\chi_{\Omega \backslash D}(x)\left(\left\langle u_{m}\right\rangle+v_{m}-h\right)\right\rangle\right), N_{j}\right)\right)=0, \quad j=1, \ldots, m, \\
& \frac{d}{d t}\left\langle u_{m}\right\rangle+\xi\left\langle u_{m}\right\rangle=-\frac{\lambda_{0}}{\operatorname{Vol}(\Omega)} \int_{\Omega \backslash D}\left(v_{m}-h\right) d x, \\
& v_{m}(0)=P_{m} u_{0}, \\
& \left\langle u_{m}(0)\right\rangle=\left\langle u_{0}\right\rangle .
\end{aligned}
$$


We can rewrite $(3.1)$ and $(3.3)$ as

$$
\begin{gathered}
\frac{\partial(\Delta)^{-1} v_{m}}{\partial t}-\varepsilon \Delta v_{m}+\frac{1}{\varepsilon}\left(f\left(\left\langle u_{m}\right\rangle+v_{m}\right)-\left\langle f\left(\left\langle u_{m}\right\rangle+v_{m}\right)\right\rangle\right) \\
+\lambda_{0}(\Delta)^{-1}\left(\chi_{\Omega \backslash D}(x)\left(\left\langle u_{m}\right\rangle+v_{m}-h\right)-\left\langle\chi_{\Omega \backslash D}(x)\left(\left\langle u_{m}\right\rangle+v_{m}-h\right)\right\rangle\right)=0, \quad \text { in } E_{m}^{\prime}, \\
v_{m}(0)=P_{m} u_{0},
\end{gathered}
$$

and we finally rewrite equation (3.1) as follows:

$$
M^{-1} \frac{d Y}{d t}+\varepsilon M Y+H(Y)=0
$$

where $M=-\left(\left(\Delta N_{i}, N_{j}\right)\right)_{i, j=1, \ldots, m}, Y=\left(\begin{array}{c}u_{m_{1}} \\ \cdot \\ \cdot \\ \cdot \\ u_{m_{m}}\end{array}\right)$, and

$$
H(Y)=\left(\begin{array}{c}
\left(\left(\frac{1}{\varepsilon}\left(f\left(\left\langle u_{m}\right\rangle+v_{m}\right)-\left\langle f\left(\left\langle u_{m}\right\rangle+v_{m}\right)\right\rangle\right)+\lambda_{0}(-\Delta)^{-1} \Xi_{m}, N_{1}\right)\right) \\
\cdot \\
\cdot \\
\cdot \\
\left(\left(\frac{1}{\varepsilon}\left(f\left(\left\langle u_{m}\right\rangle+v_{m}\right)-\left\langle f\left(\left\langle u_{m}\right\rangle+v_{m}\right)\right\rangle\right)+\lambda_{0}(-\Delta)^{-1} \Xi_{m}, N_{m}\right)\right)
\end{array}\right),
$$

where $\Xi_{m}=\chi_{\Omega \backslash D}\left(\left(\left\langle u_{m}\right\rangle+v_{m}-h\right)\right)-\left\langle\chi_{\Omega \backslash D}\left(\left(\left\langle u_{m}\right\rangle+v_{m}-h\right)\right)\right\rangle$. Note that $\left\langle u_{m}\right\rangle$ can be expressed as a function of $v_{m}$ by solving (3.2). The matrix $M$ is invertible and positive definite and $H(Y)$ depends continuously on $Y$. Applying Cauchy's theorem, we find that there exists a time $t_{m} \in(0, T)$ and a solution $Y$ to

$$
\frac{d Y}{d t}+\varepsilon M^{2} Y+M H(Y)=0
$$

on the time interval $\left[0, t_{m}\left[\right.\right.$. Having $v_{m}$, we then deduce $\left\langle u_{m}\right\rangle$ (from (3.2)).

It follows from the a priori estimates derived in the previous section for the solution $u_{m}(t)\left(u(t)\right.$ being replaced by $u_{m}(t)$; note that, in that case, these formal a priori estimates are now fully justified within the Galerkin approximation) that any local solution to (1.10) is actually a global solution defined on the whole interval $[0, T]$.

It then follows from the a priori estimates that, up to a subsequence which we do not relabel,

$$
\begin{gathered}
u_{m} \rightarrow u \text { weakly in } L^{2}\left(0, T, H^{2}(\Omega)\right), \\
\frac{\partial u_{m}}{\partial t} \rightarrow \frac{\partial u}{\partial t} \text { weakly in } L^{\frac{4}{3}}\left(0, T, W^{-2, \frac{4}{3}}(\Omega)\right),
\end{gathered}
$$

as $m \rightarrow \infty$. It follows from (3.7), (3.8), and the Aubin-Lions compactness theorem that

$$
u_{m} \rightarrow u \text { strongly in } L^{\frac{4}{3}}\left(0, T, L^{\frac{4}{3}}(\Omega)\right)
$$

and $u_{m}(x, t) \rightarrow u(x, t)$ a.e. $(x, t) \in \Omega \times[0, T]$. Moreover, since $f$ is a continuous,

$$
f\left(u_{m}(x, t)\right) \rightarrow f(u(x, t)) \text { a.e. }
$$


and, since $f\left(u_{m}\right)$ is bounded in $L^{\frac{4}{3}}\left(\Omega_{T}\right), \Omega_{T}=\Omega \times(0, T)$,

$$
f\left(u_{m}\right) \rightarrow f(u) \text { weakly in } L^{\frac{4}{3}}\left(\Omega_{T}\right),
$$

owing to the weak dominated convergence theorem. Finally, we deduce that $(-\Delta)^{-1} \frac{\partial v_{m}}{\partial t} \rightarrow$ $(-\Delta)^{-1} \frac{\partial v}{\partial t}$ weakly in $L^{\frac{4}{3}}\left(\Omega_{T}\right)$. Thus, passing to the limit in (3.5), we obtain

$$
\begin{gathered}
\frac{\partial(-\Delta)^{-1} v}{\partial t}-\varepsilon \Delta u+\frac{1}{\varepsilon}(f(u)-\langle f(u)\rangle) \\
+\lambda_{0}(-\Delta)^{-1}\left(\chi_{\Omega \backslash D}(x)(u-h)-\left\langle\chi_{\Omega \backslash D}(x)(u-h)\right\rangle\right)=0, \quad \text { in } L^{\frac{4}{3}}\left(\Omega_{T}\right) .
\end{gathered}
$$

Finally, we easily pass to the limit in (3.2), at least in a weak sense, i.e., solving explicitly this ODE.

Let now $z$ and $w$ be two solutions to (1.10) with initial data $z_{0}$ and $w_{0}$, respectively. We set $u=z-w$ and $u_{0}=z_{0}-w_{0}$ and have

$$
\begin{gathered}
\frac{\partial u}{\partial t}+\varepsilon \Delta^{2} u-\frac{1}{\varepsilon} \Delta(f(z)-f(w))+\lambda_{0} \chi_{\Omega \backslash D}(x) u=0, \quad \text { in } \Omega, \\
\frac{\partial u}{\partial \nu}=\frac{\partial \Delta u}{\partial \nu}=0, \quad \text { on } \Gamma, \\
\left.u\right|_{t=0}=u_{0}=z_{0}-w_{0}, \quad \text { in } \Omega .
\end{gathered}
$$

Integrating (3.11) over $\Omega$, we obtain

$$
\frac{d}{d t}\langle u\rangle+\lambda_{0}\left\langle\chi_{\Omega \backslash D}(x) u\right\rangle=0
$$

hence, setting again $v=u-\langle u\rangle$,

$$
\frac{\partial v}{\partial t}+\varepsilon \Delta^{2} v-\frac{1}{\varepsilon} \Delta(f(z)-f(w))+\lambda_{0}\left(\chi_{\Omega \backslash D}(x) u-\left\langle\chi_{\Omega \backslash D}(x) u\right\rangle\right)=0 .
$$

We multiply $(3.15)$ by $(-\Delta)^{-1} \bar{v}$ and find, noting that

$$
((\langle f(z)-f(w)\rangle, v))=0
$$

the differential equality

$$
\begin{aligned}
\frac{1}{2} \frac{d}{d t}\|v\|_{-1}^{2} & +\varepsilon\|\nabla v\|^{2}+\frac{1}{\varepsilon} \mathbf{R e}[((f(z)-f(w), v))] \\
& +\lambda_{0} \operatorname{Re}\left[\left(\left((-\Delta)^{-1}\left(\chi_{\Omega \backslash D}(x) u-\left\langle\chi_{\Omega \backslash D}(x) u\right\rangle\right), v\right)\right)\right]=0 .
\end{aligned}
$$

Note that 


$$
\begin{aligned}
\left|\lambda_{0}\left(\left((-\Delta)^{-1}\left(\chi_{\Omega \backslash D}(x) u-\left\langle\chi_{\Omega \backslash D}(x) u\right\rangle\right), v\right)\right)\right| & =\left|\lambda_{0}\left(\left(\chi_{\Omega \backslash D}(x) u,(-\Delta)^{-1} v\right)\right)\right| \\
& \leq c\|u\|\|v\| \\
& \leq c\left(\|v\|^{2}+|\langle u\rangle|^{2}\right),
\end{aligned}
$$

which yields, owing to Proposition 3.1,

$$
\begin{aligned}
\frac{1}{2} \frac{d}{d t}\|v\|_{-1}^{2}+\varepsilon\|\nabla v\|^{2} & +\frac{c_{0}}{\varepsilon} \int_{\Omega}\left(|u|^{4}+|z|^{2}|u|^{2}+(\operatorname{Re}[z(\bar{u})])^{2}\right) d x \\
& \leq c\left(\|z\|_{L^{4}(\Omega)}^{2}+\|w\|_{L^{4}(\Omega)}^{2}+1\right)\left(\|v\|^{2}+|\langle u\rangle|^{2}\right) .
\end{aligned}
$$

We further multiply (3.14) by $\langle\bar{u}\rangle$ and have

$$
\begin{aligned}
\frac{1}{2} \frac{d}{d t}|\langle u\rangle|^{2} & \leq \lambda_{0}\left|\left\langle\chi_{\Omega \backslash D}(x) u\right\rangle\right||\langle\bar{u}\rangle| \\
& \leq c\|u\||\langle u\rangle| \\
& \leq c\left(\|v\|^{2}+|\langle u\rangle|^{2}\right)
\end{aligned}
$$

and, recalling the interpolation inequality

$$
\|v\|^{2} \leq c\|v\|_{-1}\|\nabla v\|
$$

it follows from (3.17) and (3.18) that

$$
\begin{aligned}
\frac{d}{d t}\left(\|v\|_{-1}^{2}+|\langle u\rangle|^{2}\right)+2 \varepsilon\|\nabla v\|^{2} & +\frac{2 c_{0}}{\varepsilon} \int_{\Omega}\left(|u|^{4}+|z|^{2}|u|^{2}+(\operatorname{Re}[z(\bar{u})])^{2}\right) d x \\
& \leq c\left(\|z\|_{L^{4}(\Omega)}^{4}+\|w\|_{L^{4}(\Omega)}^{4}+1\right)\left(\|v\|_{-1}^{2}+|\langle u\rangle|^{2}\right) .
\end{aligned}
$$

We finally deduce from Gronwall's lemma that

$$
\|z(t)-w(t)\|_{H^{-1}}^{2} \leq Q\left(T,\left\|z_{0}\right\|,\left\|w_{0}\right\|\right)\left\|z_{0}-w_{0}\right\|_{H^{-1}}^{2}, \quad 0 \leq t \leq T,
$$

hence the uniqueness, as well as the continuous dependence with respect to the initial data in the $H^{-1}-$ norm.

b) Existence of the global attractor. It follows from Theorem 3.2 and (3.20) that we have the continuous (with respect to the $H^{-1}$-norm) semigroup

$$
S(t): L^{2}(\Omega) \rightarrow L^{2}(\Omega), \quad u_{0} \mapsto u(t), \quad t \geq 0
$$

(i.e. $S(0)=I, S(t+s)=S(t) \circ S(s), t, s \geq 0$ ). We then deduce from Proposition 2.12 that $S(t)$ possesses a bounded absorbing set $\mathcal{B}_{0}$ which is compact in $L^{2}(\Omega)$ and bounded in $H^{2}(\Omega)$. We thus deduce from standard results (see, e.g., [57] and [66]) the following theorem.

Theorem 3.3. The semigroup $S(t)$ possesses the connected global attractor $\mathcal{A}$ such that $\mathcal{A}$ is compact in $L^{2}(\Omega)$ and bounded in $H^{2}(\Omega)$. 
Remark 3.4. It is easy to see that we can assume, without loss of generality, that $\mathcal{B}_{0}$ is positively invariant by $S(t)$, i.e., $S(t) \mathcal{B}_{0} \subset \mathcal{B}_{0}, \forall t \geq 0$.

\section{Existence of EXPONENTIAL ATtRACTORS}

Let $z$ and $w$ be two solutions to (1.10) with initial data $z_{0}$ and $w_{0}$, respectively. We set $u=z-w$ and $u_{0}=z_{0}-w_{0}$ and have

$$
\begin{gathered}
\frac{\partial u}{\partial t}+\varepsilon \Delta^{2} u-\frac{1}{\varepsilon} \Delta(f(z)-f(w))+\lambda_{0} \chi_{\Omega \backslash D}(x) u=0, \quad \text { in } \Omega, \\
\frac{\partial u}{\partial \nu}=\frac{\partial \Delta u}{\partial \nu}=0, \quad \text { on } \Gamma \\
\left.u\right|_{t=0}=u_{0}=z_{0}-w_{0}, \quad \text { in } \Omega .
\end{gathered}
$$

Furthermore, it is sufficient here to take initial data belonging to the bounded absorbing set $\mathcal{B}_{0}$ defined in the previous section.

Lemma 4.1. Let $u=z-w$ be a solution to (4.1)-(4.3). Then, we have

$$
-\boldsymbol{R e}[((\nabla(f(z)-f(w)), \nabla u))] \leq c\|u\|_{H^{1}(\Omega)}^{2}+\varepsilon^{2}\|\Delta u\|^{2} .
$$

Proof. We first note that

$$
f(z)-f(w)=|u|^{2} u-|u|^{2} z+|z|^{2} u+2 \operatorname{Re}[z \bar{u}] z-2 \operatorname{Re}[z \bar{u}] u-u .
$$

It follows from Proposition 2.2 that

$$
\boldsymbol{\operatorname { R e }}\left[\left(\left(\nabla\left(|u|^{2} u-u\right), \nabla u\right)\right)\right] \geq-\|\nabla u\|^{2} .
$$

Besides from Proposition 2.12 and the continuous embedding $H^{2}(\Omega) \subset \mathcal{C}(\bar{\Omega})$ we have that

$$
\begin{aligned}
\mid \boldsymbol{\operatorname { R e }}\left[\left(\left(-|u|^{2} z\right.\right.\right. & \left.\left.\left.+|z|^{2} u+2 \operatorname{Re}[z \bar{u}] z-2 \operatorname{Re}[z \bar{u}] u, \Delta u\right)\right)\right] \mid \\
& \leq\left|\left(\left(-|u|^{2} z+|z|^{2} u+2 \operatorname{Re}[z \bar{u}] z-2 \operatorname{Re}[z \bar{u}] u, \Delta u\right)\right)\right| \\
& \leq 3\left\||u|^{2} z+|z|^{2} u\right\|\|\Delta u\| \\
& \leq c\left(\|u\|_{L^{4}(\Omega)}^{2}\|z\|_{L^{\infty}(\Omega)}+\|z\|_{L^{\infty}(\Omega)}^{2}\|u\|\right)\|\Delta u\| \\
& \leq c\|u\|_{H^{1}(\Omega)}^{4}+\varepsilon^{2}\|\Delta u\|^{2} .
\end{aligned}
$$

Observe now that

$$
\begin{aligned}
\|u\|_{H^{1}(\Omega)}^{4} & \leq c\left(\|z\|_{H^{1}}^{2}+\|w\|_{H^{1}}^{2}\right)\|u\|_{H^{1}}^{2} \\
& \leq c\|u\|_{H^{1}}^{2}
\end{aligned}
$$

we infer

$$
\left|\boldsymbol{\operatorname { R e }}\left[\left(\left(-|u|^{2} z+|z|^{2} u+2 \mathbf{R e}[z \bar{u}] z-2 \operatorname{Re}[z \bar{u}] u, \Delta u\right)\right)\right]\right| \leq c\|u\|_{H^{1}(\Omega)}^{2}+\varepsilon^{2}\|\Delta u\|^{2} .
$$


We finally deduce that

$$
\begin{gathered}
-\boldsymbol{\operatorname { R e }}[((\nabla(f(z)-f(w)), \nabla u))] \\
=-\operatorname{Re}\left[\left(\left(\nabla\left(|u|^{2} u-u\right), \nabla u\right)\right)\right]-\mathbf{R e}\left[\left(\left(-|u|^{2} z+|z|^{2} u+2 \mathbf{R e}[z \bar{u}] z-2 \mathbf{R e}[z \bar{u}] u, \Delta u\right)\right)\right] \\
\leq\|\nabla u\|^{2}+\left|\boldsymbol{\operatorname { R e }}\left[\left(\left(-|u|^{2} z+|z|^{2} u+2 \operatorname{Re}[z \bar{u}] z-2 \operatorname{Re}[z \bar{u}] u, \Delta u\right)\right)\right]\right| \\
\leq c\|u\|_{H^{1}(\Omega)}^{2}+\varepsilon^{2}\|\Delta u\|^{2} .
\end{gathered}
$$

Proposition 4.2. Let $u=z-w$ be a solution to (4.1)-(4.3). Then, there exist two constants $c$ and $c^{\prime}$ which only depend on $\mathcal{B}_{0}$ such that

$$
\|z-w\|^{2} \leq \frac{c}{t} e^{c^{\prime} t}\left\|z_{0}-w_{0}\right\|_{H^{-1}(\Omega)}^{2}, \quad \forall t>0 .
$$

Proof. We multiply (4.1) by tī to find

$$
\frac{1}{2} \frac{d}{d t}\left(t\|u\|^{2}\right)+\varepsilon t\|\Delta u\|^{2}+\frac{t}{\varepsilon} \mathbf{R e}[((\nabla(f(z)-f(w)), \nabla u))]+\lambda_{0} t\left\|\chi_{\Omega \backslash D}(x) u\right\|^{2}=\frac{1}{2}\|u\|^{2},
$$

which yields, owing to Lemma 4.1,

$$
\frac{d}{d t}\left(t\|u\|^{2}\right)+\lambda_{0} t\left\|\chi_{\Omega \backslash D}(x) u\right\|^{2} \leq c t\|u\|_{H^{1}(\Omega)}^{2}+\|u\|^{2} .
$$

Note that (3.19) yields

$$
\begin{aligned}
& \frac{d}{d t}\left(\|v\|_{-1}^{2}+|\langle u\rangle|^{2}\right)+2 \varepsilon\left(\|\nabla v\|^{2}+|\langle u\rangle|^{2}\right) \\
& +\frac{2 c_{0}}{\varepsilon} \int_{\Omega}\left(|u|^{4}+|z|^{2}|u|^{2}+(\operatorname{Re}[z(\bar{u})])^{2}\right) d x \leq c\left(\|v\|_{-1}^{2}+|\langle u\rangle|^{2}\right) .
\end{aligned}
$$

By using Gronwall's lemma, on account of (4.6), we find

$$
\|z(t)-w(t)\|_{H^{-1}(\Omega)}^{2} \leq c e^{c^{\prime} t}\left\|z_{0}-w_{0}\right\|_{H^{-1}(\Omega)}^{2} .
$$

Therefore, integrating $(4.6)$ over $(0, t)$, we obtain, owing to (4.7),

$$
\int_{0}^{t}\|u\|_{H^{1}(\Omega)}^{2} d s \leq c e^{c^{\prime} t}\left\|u_{0}\right\|_{H^{-1}(\Omega)}^{2} .
$$

Finally, using Gronwall's lemma, on account of (4.5), and employing (4.8) we infer

$$
\|z(t)-w(t)\|^{2} \leq \frac{c}{t} e^{c^{\prime} t}\left\|z_{0}-w_{0}\right\|_{H^{-1}(\Omega)}^{2}, \quad \forall t>0 .
$$


Proposition 4.3. The semigroup $S(t)$ is uniformly Hölder continuous on $[0, T] \times \mathcal{B}_{0}$ in the topology of $H^{-1}(\Omega)$, i.e.,

$$
\left\|S\left(t_{1}\right) z_{0}-S\left(t_{2}\right) w_{0}\right\|_{H^{-1}(\Omega)} \leq c\left(\left\|z_{0}-w_{0}\right\|_{H^{-1}(\Omega)}+\left|t_{1}-t_{2}\right|^{\frac{1}{2}}\right),
$$

where the constant $c$ only depends on $T$ and $\mathcal{B}_{0}$.

Proof. The Lipschitz continuity with respect to the initial data is an immediate corollary of (3.20). Actually, it suffices to prove the Hölder continuity with respect to time. We have

$$
\begin{aligned}
\left\|u\left(t_{1}\right)-u\left(t_{2}\right)\right\|_{H^{-1}(\Omega)} & =\left\|\int_{t_{1}}^{t_{2}} \frac{\partial u}{\partial t} d \tau\right\|_{H^{-1}(\Omega)} \\
& \leq\left|\int_{t_{1}}^{t_{2}} \| \frac{\partial u}{\partial t}\right|_{H^{-1}(\Omega)} d \tau \mid \\
& \leq\left|t_{1}-t_{2}\right|^{\frac{1}{2}}\left|\int_{t_{1}}^{t_{2}}\left\|\frac{\partial u}{\partial t}\right\|_{H^{-1}(\Omega)}^{2} d \tau\right|^{\frac{1}{2}} .
\end{aligned}
$$

Multiplying (2.3) by $\frac{\partial \bar{v}}{\partial t}$, we find, noting that $\left\langle\frac{\partial \bar{v}}{\partial t}\right\rangle=0$,

$$
\begin{array}{r}
\frac{\varepsilon}{2} \frac{d}{d t}\|\nabla u\|^{2}+\left\|\frac{\partial v}{\partial t}\right\|_{-1}^{2}+\frac{1}{\varepsilon} \mathbf{R e}\left[\left(\left(f(u), \frac{\partial v}{\partial t}\right)\right)\right] \\
+\lambda_{0} \operatorname{Re}\left[\left(\left(\chi_{\Omega \backslash D}(x)(u-h),(-\Delta)^{-1} \frac{\partial v}{\partial t}\right)\right)\right]=0 .
\end{array}
$$

Here,

$$
\begin{gathered}
\left|\lambda_{0} \mathbf{R e}\left[\left(\left(\chi_{\Omega \backslash D}(x)(u-h),(-\Delta)^{-1} \frac{\partial v}{\partial t}\right)\right)\right]\right| \\
\quad=\left|\lambda_{0}\left(\left(\chi_{\Omega \backslash D}(x)(u-h),(-\Delta)^{-1} \frac{\partial v}{\partial t}\right)\right)\right| \\
\leq c\|u-h\|\left|\left\|\frac{\partial v}{\partial t}\right\|\right|_{-1} \\
\quad \leq c\left(\|v\|^{2}+|\langle u\rangle|^{2}+\|h\|^{2}\right)+\frac{1}{4}\left\|\frac{\partial v}{\partial t}\right\|_{-1}^{2}
\end{gathered}
$$

Furthermore,

$$
\left|\frac{1}{\varepsilon} \operatorname{Re}\left[\left(\left(f(u), \frac{\partial v}{\partial t}\right)\right)\right]\right| \leq c\|\nabla f(u)\||| \frac{\partial v}{\partial t} \|\left.\right|_{-1}
$$

which yields

$$
\varepsilon \frac{d}{d t}\|\nabla u\|^{2}+\left\|\frac{\partial v}{\partial t}\right\|_{-1}^{2} \leq c\left(\|v\|^{2}+|\langle u\rangle|^{2}+\|h\|^{2}\right)+c^{\prime}\|\nabla f(u)\|^{2} .
$$


From (3.14), we have that

$$
\left\langle\frac{\partial u}{\partial t}\right\rangle \leq c\|u\|
$$

and, (4.12) can be written as

$$
\varepsilon \frac{d}{d t}\|\nabla u\|^{2}+\left\|\frac{\partial v}{\partial t}\right\|_{-1}^{2}+\left\langle\frac{\partial u}{\partial t}\right\rangle^{2} \leq c\left(\|v\|^{2}+|\langle u\rangle|^{2}+\|h\|^{2}\right)+c^{\prime}\|\nabla f(u)\|^{2} .
$$

Note that

$$
\begin{aligned}
\|\nabla f(u)\| & =\left\|f^{\prime}(u) \nabla u\right\| \\
& \leq Q\left(\|u\|_{L^{\infty}(\Omega)}\right)\|\nabla u\| \\
& \leq c\|\nabla u\|,
\end{aligned}
$$

owing to the definition of $\mathcal{B}_{0}$ and the continuous embedding $H^{2}(\Omega) \subset \mathcal{C}(\bar{\Omega})$. We then deduce from (2.18) and (4.13) that

$$
\int_{t_{1}}^{t_{2}}\left\|\frac{\partial u}{\partial t}\right\|_{H^{-1}(\Omega)}^{2} \leq c
$$

where the constant $c$ only depends on $\mathcal{B}_{0}$ and $T$ such that $t_{1}, t_{2} \in[0, T]$, so that (4.10) yields

$$
\left\|u\left(t_{1}\right)-u\left(t_{2}\right)\right\|_{H^{-1}(\Omega)} \leq c\left|t_{1}-t_{2}\right|^{\frac{1}{2}},
$$

where the constant $c$ only depends on $\mathcal{B}_{0}$ and $T$ such that $t_{1}, t_{2} \in[0, T]$.

We finally deduce from Theorem 3.2, Proposition 4.2, and Proposition 4.3 the following result (see, e.g., [26] and [27]).

Theorem 4.4. The semigroup $S(t)$ possesses an exponential attractor $\mathcal{M} \subset \mathcal{B}_{0}$, i.e.,

(i) $\mathcal{M}$ is compact in $H^{-1}(\Omega)$;

(ii) $\mathcal{M}$ is positively invariant, $S(t) \mathcal{M} \subset \mathcal{M}, \forall t \geq 0$;

(iii) $\mathcal{M}$ has finite fractal dimension in $H^{-1}(\Omega)$;

(iii) $\mathcal{M}$ attracts exponentially fast the bounded subsets of $L^{2}(\Omega)$,

$$
\begin{gathered}
\forall B \subset L^{2}(\Omega) \text { bounded, } \operatorname{dist}_{H^{-1}(\Omega)}(S(t) B, \mathcal{M}) \leq Q\left(\|B\|_{L^{2}(\Omega)}\right) e^{-\xi t}, \\
\xi>0, t \geq 0,
\end{gathered}
$$

where the constant $\xi$ is independent of $B$ and dist $H_{H^{-1}(\Omega)}$ denotes the Hausdorff semidistance between sets defined by

$$
\operatorname{dist}_{H^{-1}(\Omega)}(A, B)=\sup _{a \in A} \inf _{b \in B}\|a-b\|_{H^{-1}(\Omega)} .
$$

Remark 4.5. Setting $\tilde{\mathcal{M}}=S(1) \mathcal{M}$, we can prove that $\tilde{\mathcal{M}}$ is an exponential attractor for $S(t)$, but now in the topology of $L^{2}(\Omega)$ (see, e.g., [28]). 
Since $\mathcal{M}$ (or $\tilde{\mathcal{M}}$ ) is a compact attracting set, we deduce from Theorem 4.4 the following corollary.

Corollary 4.6. The semigroup $S(t)$ possesses the finite-dimensional global attractor $\mathcal{A} \subset$ $\mathcal{B}_{0}$.

Remark 4.7. i) We can also follow [6] to prove the continuity of the isophote vectors at the boundary of the inpainting domain for problem (1.10) when $\lambda_{0}$ is large.

ii) An important particular case of problem (1.10) arises when the initial imaginary part vanishes (i.e., $\left.u_{2}(0, x)=0\right)$, meaning that the damaged image (binary image in that case) has two values, -1 and 1 . In that case, numerical simulations (we have not been able to prove it) show that the imaginary part of the solution remains zero for all times.

iii) We studied the (global) dynamics of the system when $\lambda_{0}$ and $\varepsilon$ are constants. More generally, all constants here and in the previous sections depend on $\lambda_{0}$ and $\varepsilon$ and grow as these quantities go to $+\infty$ and 0 , respectively. In particular, the dependence on $\lambda_{0}$ is an important issue in view of inpainting applications (see [6]) and a natural question is whether one can have an upper bound on the dimension of the global attractor which is independent of this quantity (here, the upper bound that we obtain explodes as $\lambda_{0}$ goes to $+\infty$ ). A natural (but involved, see, e.g., [66]) problem would be to find a lower bound on this dimension (possibly, in terms of $\lambda_{0}$ ). This will be investigated elsewhere.

\section{NumeriCAL Simulations}

The author in [34] and [35] proposed a convexity splitting scheme for gradient flowderived equations which results in an unconditionally gradient stable time discretization scheme. In particular, the scheme is stable for any arbitrarily large time step. Here, the idea consists in dividing the energy functional into two parts, a convex one and a concave one. Then, the convex part is treated implicitly, while the concave one is treated explicitly. This scheme has been used as a specific fast solver for the Bertozzi-EsedogluGillette-Cahn-Hilliard equation, see [5], [6], [7], and [10].

As in the case of the Bertozzi-Esedoglu-Gillette-Cahn-Hilliard equation, (1.10) is not a gradient flow. However, it is the sum of a gradient descent with respect to the $H^{-1}$-inner product for the original Cahn-Hilliard energy and a gradient descent with respect to the $L^{2}$-inner product for the additional fidelity energy. We recall that the complex CahnHilliard energy is given by

$$
\mathcal{E}(u)=\int_{\Omega}\left(\frac{\varepsilon}{2}|\nabla u|^{2}+\frac{1}{\varepsilon}\left(\frac{1}{4}|u|^{4}-\frac{1}{2}|u|^{2}\right)\right) d x
$$

while the additional fidelity energy reads

$$
\mathcal{E}^{\prime}(u)=\frac{\lambda_{0}}{2} \int_{\Omega \backslash D}|u-h|^{2} d x .
$$

In view of this, we write $\mathcal{E}(u)=\mathcal{E}_{1}(u)-\mathcal{E}_{2}(u)$ and $\mathcal{E}^{\prime}(u)=\mathcal{E}_{1}^{\prime}(u)-\mathcal{E}_{2}^{\prime}(u)$, where

$$
\mathcal{E}_{1}(u)=\int_{\Omega}\left(\frac{\varepsilon}{2}|\nabla u|^{2}+\frac{c_{1}}{2}|u|^{2}\right) d x
$$


and

$$
\begin{gathered}
\mathcal{E}_{2}(u)=\int_{\Omega}\left(-\frac{1}{\varepsilon}\left(\frac{1}{4}|u|^{4}-\frac{1}{2}|u|^{2}\right)+\frac{c_{1}}{2}|u|^{2}\right) d x, \\
\mathcal{E}_{1}^{\prime}(u)=\int_{\Omega} \frac{c_{2}}{2}|u|^{2} d x
\end{gathered}
$$

$$
\mathcal{E}_{2}^{\prime}(u)=\int_{\Omega}\left(-\frac{\lambda}{2}|u-h|^{2}+\frac{c_{2}}{2}|u|^{2}\right) d x
$$

$c_{1}$ and $c_{2}$ being nonnegative constants which need to be chosen large enough so that the energies $\mathcal{E}_{1}(u), \mathcal{E}_{2}(u), \mathcal{E}_{1}^{\prime}(u)$, and $\mathcal{E}_{2}^{\prime}(u)$ are convex.

As far as the Euler time discretization for this problem is concerned, the time step $\delta t>0$ is fixed. The resulting time-stepping scheme reads

$$
\frac{u_{h}^{n+1}-u_{h}^{n}}{\delta t}=-\nabla_{H^{-1}}\left(\mathcal{E}_{1}\left(u_{h}^{n+1}\right)-\mathcal{E}_{2}\left(u_{h}^{n}\right)\right)-\nabla_{L^{2}}\left(\mathcal{E}_{1}^{\prime}\left(u_{h}^{n+1}\right)-\mathcal{E}_{2}^{\prime}\left(u_{h}^{n}\right)\right),
$$

where $\nabla_{H^{-1}}$ and $\nabla_{L^{2}}$ denote the gradient descents with respect to the $H^{-1}-$ and $L^{2}$-inner products, respectively. This translates into a numerical scheme of the form

$\frac{u_{h}^{n+1}-u_{h}^{n}}{\delta t}+\varepsilon \Delta^{2} u_{h}^{n+1}-c_{1} \Delta u_{h}^{n+1}+c_{2} u_{h}^{n+1}=\frac{1}{\varepsilon} \Delta f\left(u_{h}^{n}\right)+\lambda_{0}\left(\chi_{\Omega \backslash D}(x)\left(h-u_{h}^{n}\right)\right)-c_{1} \Delta u_{h}^{n}+c_{2} u_{h}^{n}$.

Therefore, using the operator splitting (see [22], [30], [38], and [44]), the final scheme reads

$$
\begin{aligned}
& \frac{1}{\delta t}\left(\left(u_{h}^{n+1}, \phi\right)\right)+\left(\left(\nabla \mu_{h}^{n+1}, \nabla \phi\right)\right)+c_{1}\left(\left(\nabla u_{h}^{n+1}, \nabla \phi\right)\right)+c_{2}\left(\left(u_{h}^{n+1}, \phi\right)\right) \\
&= \frac{1}{\delta t}\left(\left(u_{h}^{n}, \phi\right)\right)+\lambda_{0}\left(\left(\chi_{\Omega \backslash D}(x)\left(h(x)-u_{h}^{n}\right), \phi\right)\right) \\
&+c_{1}\left(\left(\nabla u_{h}^{n}, \nabla \phi\right)\right)+c_{2}\left(\left(u_{h}^{n}, \phi\right)\right), \quad \forall \phi \in H^{1}(\Omega), \\
&\left(\left(\mu_{h}^{n+1}, \psi\right)\right)-\varepsilon\left(\left(\nabla u_{h}^{n+1}, \nabla \psi\right)\right)=\frac{1}{\varepsilon}\left(\left(f\left(u_{h}^{n}\right), \psi\right)\right), \quad \forall \psi \in H^{1}(\Omega) .
\end{aligned}
$$

In this scheme, we use a $P 1$-finite element for the space discretization. The numerical simulations are performed with the software Freefem ++ (see [43]).

Having this convexity splitting in mind, we follow the dynamic two-steps numerical algorithm proposed in [5] and [6] to obtain good inpainting results. The idea here is that the edges in the image should be connected with a large value of the diffuse interface thickness $\varepsilon$ and then, switching the interface diffuse thickness $\varepsilon$ to a smaller value (which depends on the mesh discretization), we obtain the final inpainting result. In the grayscale inpainting tests below, we further use the information outside the inpainting domain in the given image to obtain better inpainting results.

Binary image test. The gray region in Figure 1(a) corresponds to the inpainting region. We run the complex version of the modified Cahn-Hilliard equation with $\varepsilon=0.1$. We are close to a steady state at $t=592$, as shown in Figure 1(b) (real part of the solution which is equal to the solution, see Remark 4.7 (ii)). Then, we take $\varepsilon=0.001$ and run again the complex version of the modified Cahn-Hilliard equation, taking Figure 1(b) as an initial datum. We are close to a steady state at $t=677$, as shown in Figure 1(c) (real part of the solution which is equal to the solution, see Remark 4.7 (ii)). In this test, $\lambda_{0}=90000$, $\delta t=1, c_{1}=3000$, and $c_{2}=270000$. Furthermore, $\Omega$ is a $(0,0.5) \times(0,0.5)$-square. The 


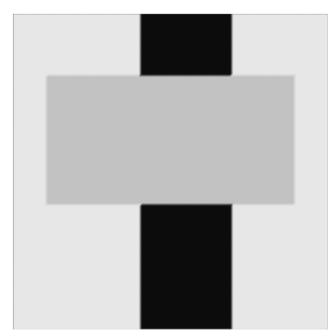

(a)

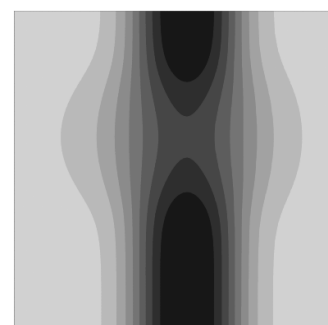

(b)

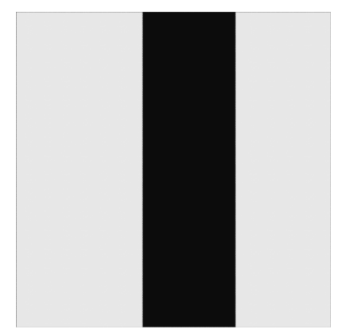

(c)

Figure 1. (a) Inpainting region in gray. (b) Solution at $t=592, \varepsilon=0.1$.

(c) Solution at $t=677, \varepsilon=0.001$.

triangulation is obtained by dividing it into $100 \times 100$ rectangles and by dividing each rectangle along the same diagonal.

Grayscale image tests. Figure 2 shows the efficiency of our proposed model in disocclusion applications (disocclusion means the recovery of hidden parts of objects in an image by interpolation from the neighborhood of the occluded area; for more details, see, e.g., [54]). Figure 2(a) and Figure 2(b) correspond to the damaged image and the masked part, respectively. We then run the complex version of the modified Cahn-Hilliard equation with a large value of the diffuse interface thickness $(\varepsilon=0.1)$. We are close to a steady state at $t=99$, as shown in Figure 2(c) (real part), and switch $\varepsilon$ to a smaller value $(\varepsilon=0.001)$. We are close to a steady state at $t=875$, as shown in Figure $2(\mathrm{~d})$ (real part) and in Figure 2(e) (imaginary part). Finally, we use the information on the image in $\Omega \backslash D$ given in the damaged image $2(\mathrm{a})$ in the inpainting result $2(\mathrm{~d})$ to obtain the final inpainting result in Figure 2(f). Here, $\lambda_{0}=100000, \delta t=1, c_{1}=3000$, and $c_{2}=300000$.

Figure 3 shows the efficiency of our proposed model to remove texts. Figure 3(a) and Figure 3(b) correspond to the damaged image and the masked part, respectively. As above, we first run the complex version of the modified Cahn-Hilliard equation with a large value of the diffuse interface thickness $(\varepsilon=0.1)$. We are close to a steady state at $t=273$ and then switch $\varepsilon$ to a smaller value $(\varepsilon=0.001)$. We are close to a steady state at $t=2265$, as shown in Figure 3(c). Finally, we use the information on the image outside $D$ given in the damaged image 3(a) in the inpainting result 3(c) to obtain the final inpainting result in Figure $3(\mathrm{~d})$. Here, $\lambda_{0}=10000, \delta t=1, c_{1}=3000$, and $c_{2}=30000$.

In our final test, Figure 4(a) and Figure 4(b) correspond to the damaged image and the masked part, respectively. As above, we first run the complex version of the modified Cahn-Hilliard equation with a large value of the diffuse interface thickness $(\varepsilon=0.1)$. We are close to a steady state at $t=115$ and then switch $\varepsilon$ to a smaller value $(\varepsilon=0.001)$. We are close to a steady state at $t=1162$, as shown in Figure $4(\mathrm{c})$. Finally, we use the information on the image in $\Omega \backslash D$ given in the damaged image 4(a) in the inpainting result $4(\mathrm{c})$ to obtain the final inpainting result in Figure $4(\mathrm{~d})$. Here, $\lambda_{0}=100000, \delta t=1$, $c_{1}=3000$, and $c_{2}=300000$. 


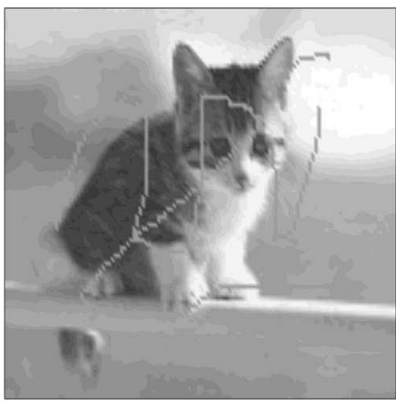

(a)

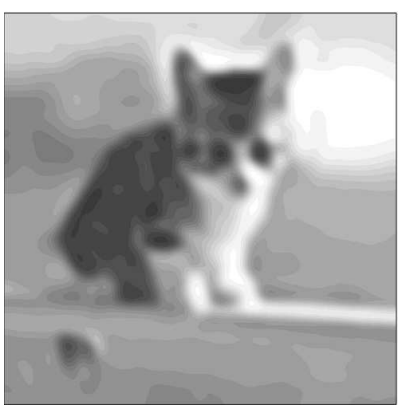

(d)

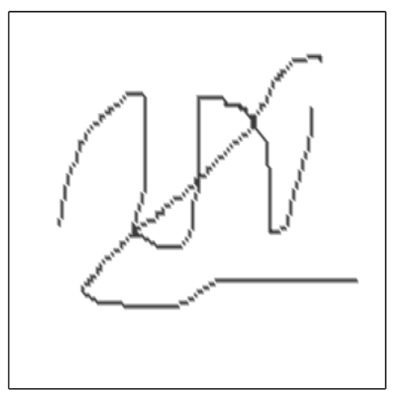

(b)

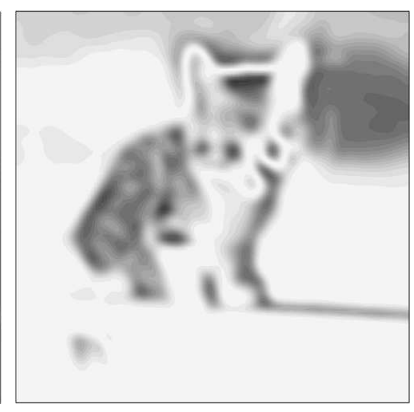

(e)

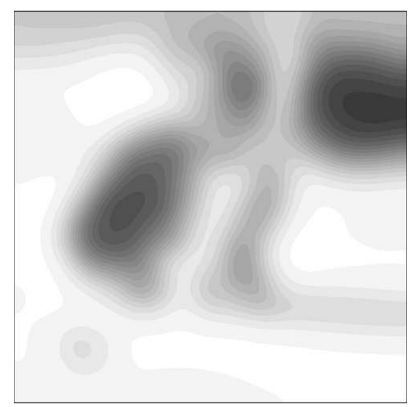

(c)

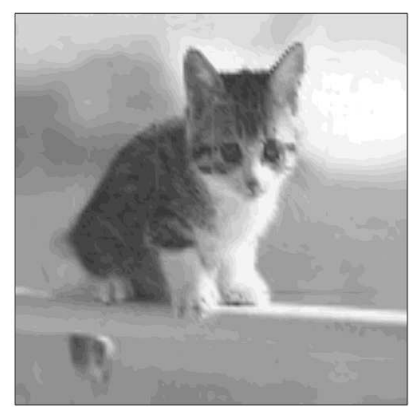

(f)

Figure 2. (a) Damaged image $(128 \times 102$ pixels $)$. (b) Masked part. (c) Real part of the solution at $t=99$ with $\varepsilon=0.1$. (d) Real part of the solution at $t=875$ with $\varepsilon=0.001$. (e) Imaginary part of the solution at $t=875$ with $\varepsilon=0.001$. (f) Using the information of the image outside $D$ given in (a) in the inpainting result (d).

Conclusion. We proposed in this article a complex version of the Bertozzi-EsedogluGillette-Cahn-Hilliard equation, introduced for binary image inpainting, in view of applications to grayscale image inpainting. Though the equation has the same structure as the binary model, we had, owing to the fact that the order parameter is now complex valued, to derive proper and careful estimates on the nonlinear term. In particular, we proved the existence and uniqueness of solutions, as well as the existence of the finite-dimensional global attractor. Furthermore, we obtained numerical simulations which show the efficiency of the model.

\section{REFERENCES}

[1] C. Ballester, M. Bertalmio, V. Caselles, G. Sapiro, and J. Verdera, Filling-in by joint interpolation of vector fields and grey levels, IEEE Trans. Imag. Proc. 10 (2001), 1200-1211.

[2] Z. Belhachmi, M. Kallel, M. Moakher, and A. Theljani, Weighted Harmonic and Ginzburg-Landau equations in image inpainting, preprint.

[3] M. Bertalmio, A. Bertozzi, and G. Sapiro, Navier-Stokes, fluid dynamics, and image and video inpainting, in Proccedings of the IEEE computer vision and pattern recognition, 335-362, 2001.

[4] M. Bertalmio, G. Sapiro, V. Casselles, and C. Ballester, Image inpainting, in Siggraph 2000, Computer Graphics Proccedings, K. Akeley ed., ACM Press/Addison-Wesley, New York, 417-424, 2000. 


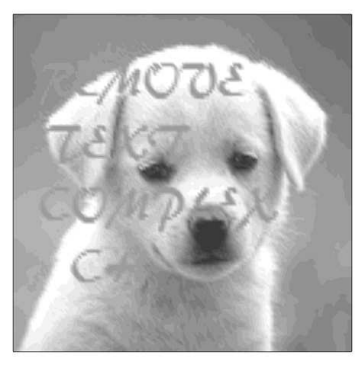

(a)

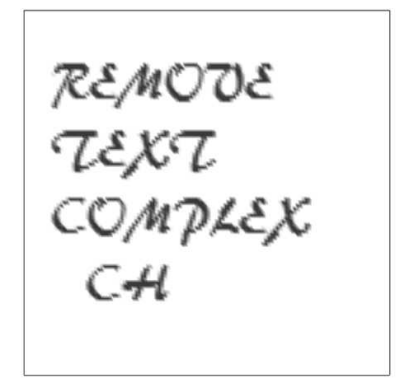

(b)

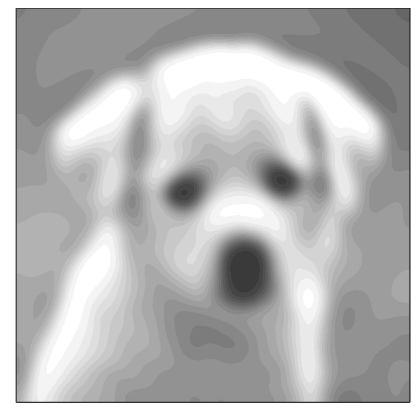

(c)

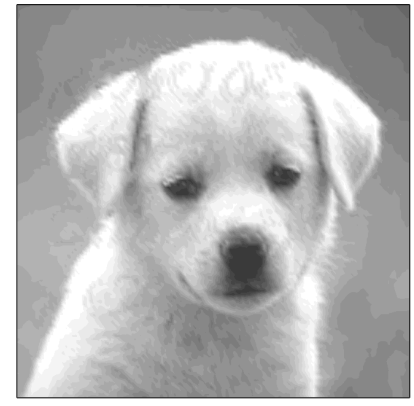

(d)

Figure 3. (a) Damaged image $(128 \times 102$ pixels). (b) Masked part. (c) Real part of the solution. (d) Using the information of the image outside $D$ given in (a) in the inpainting result (c).

[5] A. Bertozzi, S. Esedoglu, and A. Gillette, Inpainting of binary images using the Cahn-Hilliard equation, IEEE Trans. Imag. Proc. 16 (2007), 285-291.

[6] A. Bertozzi, S. Esedoglu, and A. Gillette, Analysis of a two-scale Cahn-Hilliard model for binary image inpainting, Multiscale Model. Simul. 6 (2007), 913-936.

[7] J. Bosch, D. Kay, M. Stoll, and A.J. Wathen, Fast solvers for Cahn-Hilliard inpainting, SIAM J. Imag. Sci. 7 (2013), 67-97.

[8] J. Bosch and M. Stoll, A fractional inpainting model based on the vector-valued Cahn-Hilliard equation, SIAM J. Imag. Sci., to appear.

[9] C. Braverman, Photoshop retouching handbook, IDG Books Worldwide, Amsterdam, 1998.

[10] M. Burger, L. He, and C. Schönlieb, Cahn-Hilliard inpainting and a generalization for grayvalue images, SIAM J. Imag. Sci. 3 (2009), 1129-1167.

[11] J.W. Cahn, On spinodal decomposition, Acta Metall. 9 (1961), 795-801.

[12] J.W. Cahn and J.E. Hilliard, Free energy of a nonuniform system I. Interfacial free energy, J. Chem. Phys. 28 (1958), 258-267.

[13] V. Chalupeckí, Numerical studies of Cahn-Hilliard equations and applications in image processing, in Proceedings of Czech-Japanese Seminar in Applied Mathematics 2004 (August 4-7, 2004), Czech Technical University in Prague.

[14] T.F. Chan and J. Shen, Mathematical models of local non-texture inpaintings, SIAM J. Appl. Math. 62 (2001), 1019-1043.

[15] T.F. Chan, J. Shen, and S. Kang, Non-texture inpainting by curvature-driven diffusions, J. Visual Commun. Imag. Represenation 12 (2001), 436-449.

[16] T.F. Chan and J. Shen, Euler's elastica and curvature-based image inpainting, SIAM J. Appl. Math. 63 (2002), 564-592. 


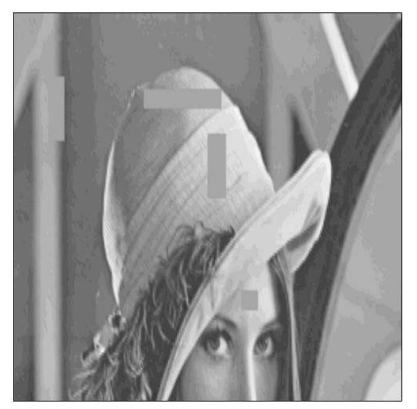

(a)

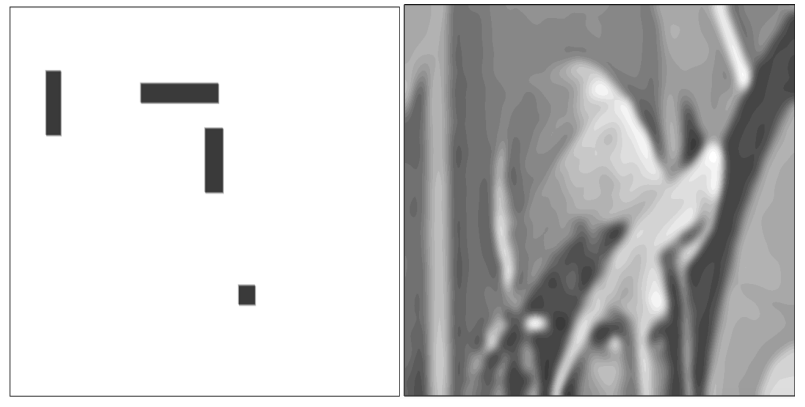

(b)

(c)

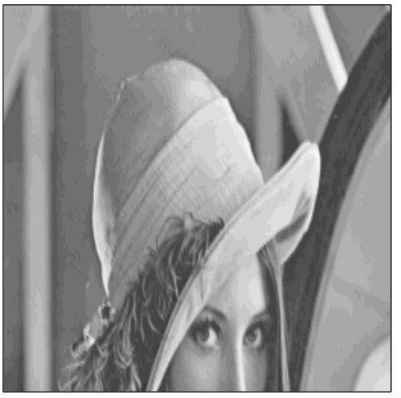

(d)

Figure 4. (a) Damaged image $(256 \times 256$ pixels $)$. (b) Masked part. (c) Real part of the solution. (d) Using the information of the image outside $D$ given in (a) in the inpainting result (c).

[17] L. Cherfils, H. Fakih, and A. Miranville, Finite-dimensional attractors for the Bertozzi-EsedogluGillette-Cahn-Hilliard equation in image inpainting, Inv. Prob. Imag. 9 (2015), 105-125.

[18] L. Cherfils, H. Fakih, and A. Miranville, On the Bertozzi-Esedoglu-Gillette-Cahn-Hilliard equation with logarithmic nonlinear terms, SIAM J. Imag. Sci. 8 (2015), 1123-1140.

[19] L. Cherfils, H. Fakih, and A. Miranville, A Cahn-Hilliard system with a fidelity term for color image inpainting, J. Math. Imag. Vision, to appear.

[20] L. Cherfils, A. Miranville, and S. Zelik, The Cahn-Hilliard equation with logarithmic potentials, Milan J. Math. 79 (2011), 561-596.

[21] L. Cherfils, A. Miranville, and S. Zelik, On a generalized Cahn-Hilliard equation with biological applications, Discrete Cont. Dyn. Systems B 19 (2014), 2013-2026.

[22] L. Cherfils, M. Petcu, and M. Pierre, A numerical analysis of the Cahn-Hilliard equation with dynamic boundary conditions, Discrete Cont. Dyn. Systems 27 (2010), 1511-1533.

[23] D. Cohen and J.M. Murray, A generalized diffusion model for growth and dispersion in a population, J. Math. Biol. 12 (1981), 237-248.

[24] F. Della Porta and M. Grasselli, Convective nonlocal Cahn-Hilliard equations with reaction terms, Discrete Cont. Dyn. Systems B 20 (2015), 1529-1553.

[25] I.C. Dolcetta, S.F. Vita, and R. March, Area-preserving curve-shortening flows: from phase separation to image processing, Interfaces Free Bound. 4 (2002), 325-343.

[26] A. Eden, C. Foias, B. Nicolaenko, and R. Temam, Exponential attractors for dissipative evolution equations, Research in Applied Mathematics, Vol. 37, John-Wiley, New York, 1994.

[27] M. Efendiev, A. Miranville, and S. Zelik, Exponential attractors for a nonlinear reaction-diffusion system in $R^{3}$, C.R. Acad. Sci. Paris Série I Math. 330 (2000), 713-718. 
[28] M. Efendiev, A. Miranville, and S. Zelik, Exponential attractors for a singularly perturbed CahnHilliard system, Math. Nach. 272 (2004), 11-31.

[29] C.M. Elliott, The Cahn-Hilliard model for the kinetics of phase separation, in Mathematical models for phase change problems, J.F. Rodrigues ed., International Series of Numerical Mathematics, Vol. 88, Birkhäuser, Basel, 1989.

[30] C.M. Elliott, D.A. French, and F.A. Milner, A second order splitting method for the Cahn-Hilliard equation, Numer. Math. 54 (1989), 575-590.

[31] G. Emile-Male, The restorer's handbook of easel painting, Van Nostrand Reinold, 1976.

[32] S. Esedoglu and R. March, Segmentation with depth but without detecting junctions, J. Math. Imag. Vision 18 (2003), 7-15.

[33] S. Esedoglu and J. Shen, Digital inpainting based on the Mumford-Shah-Euler image model, Eur. J. Appl. Math. 2000 (2000), 1-26.

[34] D.J. Eyre, An unconditionally stable one-step scheme for gradient systems, Technical report, Department of Mathematics, University of Utah, Salt Lake City, Utah, USA, 1997.

[35] D.J. Eyre, Unconditionally gradient stable time marching the Cahn-Hilliard equation, Mater. Res. Soc. Proceedings, Vol. 529, J.W. Bullard, L.Q. Chen, R.K. Kalia, and A.M. Stoneham eds., Cambridge University Press, 39-46, 1998.

[36] H. Fakih, A Cahn-Hilliard equation with a proliferation term for biological and chemical applications, Asympt. Anal. 94 (2015), 71-104.

[37] P.C. Fife, Models for phase separation and their mathematics, Electron. J. Diff. Eqns. 13 (2002), $353-370$.

[38] M. Grasselli and M. Pierre, A splitting method for the Cahn-Hilliard equation with inertial term, Math. Models Methods Appl. Sci. 20 (2010), 1-28.

[39] J.B. Greer and A.L. Bertozzi, $H^{1}$ solutions of a class of fourth order nonlinear equations for image processing, Discrete Cont. Dyn. Systems 10 (2004), 349-366.

[40] J.B. Greer and A.L. Bertozzi, Traveling wave solutions of fourth order PDE's for image processing, SIAM J. Math. Anal. 36 (2004), 38-68.

[41] J.B. Greer, A.L. Bertozzi, and G. Sapiro, Fourth order partial differential equations on general geometries, J. Comput. Phys. 216 (2006), 216-246.

[42] H. Grossauer and O. Scherzer, Using the Complex Ginzburg-Landau equation for digital inpainting in $2 D$ and $3 D$, Scale Space Methods in Computer Vision, Lecture Notes in Computer Science 2695, 225-236, 2003.

[43] F. Hecht, New development in FreeFem++, J. Numer. Math. 20 (2012), 251-265.

[44] S. Injrou and M. Pierre, Stable discretizations of the Cahn-Hilliard-Gurtin equations, Discrete Cont. Dyn. Systems 22 (2008), 1065-1080.

[45] E. Khain and L.M. Sander, A generalized Cahn-Hilliard equation for biological applications, Phys. Rev. E 77 (2008), 051129.

[46] D. King, The Commissar vanishes, Henry Holt and Company, 1997.

[47] I. Klapper and J. Dockery, Role of cohesion in the material description of biofilms, Phys. Rev. E $\mathbf{7 4}$ (2006), 0319021.

[48] R.V. Kohn and F. Otto, Upper bounds for coarsening rates, Commun. Math. Phys. 229 (2002), $375-395$.

[49] A.C. Kokaram, Motion Picture Restoration: Digital Algorithms for Artefact Suppression in Degraded Motion Picture Film and Video, Springer Verlag, 1998.

[50] J.S. Langer, Theory of spinodal decomposition in alloys, Ann. Phys. 65 (1975), 53-86.

[51] Q.-X. Liu, A. Doelman, V. Rottschäfer, M. de Jager, P.M.J. Herman, M. Rietkerk, and J. van de Koppel, Phase separation explains a new class of self-organized spatial patterns in ecological systems, Proc. Natl. Acad. Sci. 110 (2013), 11905-11910; also available online at http://www.pnas.org/cgi/doi/10.1073/pnas.1222339110.

[52] S. Maier-Paape and T. Wanner, Spinodal decomposition for the Cahn-Hilliard equation in higher dimensions. Part I: Probability and wavelength estimate, Commun. Math. Phys. 195 (1998), 435464. 
[53] S. Maier-Paape and T. Wanner, Spinodal decomposition for the Cahn-Hilliard equation in higher dimensions: Nonlinear dynamics, Arch. Ration. Mech. Anal. 151 (2000), 187-219.

[54] S. Masnou and J.M. Morel, Level lines based disocclution, in Proceedings of the 5th IEEE International Conference on Image Processing, Vol. 3, 259-263, 1998.

[55] A. Miranville, Asymptotic behavior of a generalized Cahn-Hilliard equation with a proliferation term, Appl. Anal. 92 (2013), 1308-1321.

[56] A. Miranville, A generalized Cahn-Hilliard equation with logarithmic potentials, in Continuous and Distributed Systems II, Springer, 137-148, 2015.

[57] A. Miranville and S. Zelik, Attractors for dissipative partial differential equations in bounded and unbounded domains, in Handbook of Differential Equations, Evolutionary Partial Differential Equations, Vol. 4, C.M. Dafermos and M. Pokorny eds., Elsevier, Amsterdam, 103-200, 2008.

[58] B. Nicolaenko, B. Scheurer, and R. Temam, Some global dynamical properties of a class of pattern formation equations, Commun. Partial Diff. Eqns. 14 (1989), 245-297.

[59] A. Novick-Cohen, The Cahn-Hilliard equation: Mathematical and modeling perspectives, Adv. Math. Sci. Appl. 8 (1998), 965-985.

[60] A. Novick-Cohen, The Cahn-Hilliard equation, in Handbook of Differential Equations, Evolutionary Partial Differential Equations, Vol. 4, C.M. Dafermos and M. Pokorny eds., Elsevier, Amsterdam, 201-228, 2008.

[61] A. Oron, S.H. Davis, and S.G. Bankoff, Long-scale evolution of thin liquid films, Rev. Mod. Phys. 69 (1997), 931-980.

[62] P. Perona and J. Malik, Scale-space and edge detection using anistropic diffusion, IEEE Trans. Pattern Anal. Mach. Intell. 12 (1990), 629-639.

[63] L. Rudin, S. Osher, and E. Fatemi, Nonlinear total variation based noise removal algorithms, Phys. D 60 (1992), 259-268.

[64] C.-B. Schönlieb and A. Bertozzi, Unconditionally stable schemes for higher order inpainting, Commun. Math. Sci. 9 (2011), 413-457.

[65] J. Shen, Inpainting and the fundamental problem of image processing, SIAM News 36, 1997.

[66] R. Temam, Infinite-dimensional dynamical systems in mechanics and physics, Second edition, Applied Mathematical Sciences, Vol. 68, Springer-Verlag, New York, 1997.

[67] U. Thiele and E. Knobloch, Thin liquid films on a slightly inclined heated plate, Phys. D 190 (2004), 213-248.

[68] S. Tremaine, On the origin of irregular structure in Saturn's rings, Astron. J. 125 (2003), 894-901.

${ }^{1}$ Université DE LA ROCHELle

Laboratoire Mathématiques, Image et Applications

Avenue Michel Crépeau

F-17042 La Rochelle Cedex, France

E-mail address: laurence.cherfils@univ-lr.fr

${ }^{2}$ Université de Poitiers

Laboratoire de Mathématiques et Applications

UMR CNRS 7348 - SP2MI

Boulevard Marie et Pierre Curie - Téléport 2

F-86962 Chasseneull Futuroscope Cedex, France

E-mail address: Hussein.Fakih@math.univ-poitiers.fr

E-mail address: Alain.Miranville@math.univ-poitiers.fr 